\title{
Random Polycrystals of Grains Containing Cracks: Model of Quasistatic Elastic Behavior for Fractured Systems
}

\author{
James G. Berryman ${ }^{1, *}$ and Vladimir Grechka ${ }^{2, \dagger}$ \\ ${ }^{1}$ University of California, Lawrence Berkeley National Laboratory, \\ 1 Cyclotron Road MS 90R1116, Berkeley, CA 94720, USA \\ ${ }^{2}$ Shell International Exploration and Production, Inc., \\ 3737 Bellaire Blvd., P. O. Box 481, Houston, TX 7ro01-0481
}

(Dated: July 18, 2006)

\begin{abstract}
A model study on fractured systems was performed using a concept that treats isotropic cracked systems as ensembles of cracked grains by analogy to isotropic polycrystalline elastic media. The approach has two advantages: (a) Averaging performed is ensemble averaging, thus avoiding the criticism legitimately leveled at most effective medium theories of quasistatic elastic behavior for cracked media based on volume concentrations of inclusions. Since crack effects are largely independent of the volume they occupy in the composite, such a non-volume-based method offers an appealingly simple modeling alternative. (b) The second advantage is that both polycrystals and fractured media are stiffer than might otherwise be expected, due to natural bridging effects of the strong components. These same effects have also often been interpreted as crack-crack screening in high-crack-density fractured media, but there is no inherent conflict between these two interpretations of this phenomenon. Results of the study are somewhat mixed. The spread in elastic constants observed in a set of numerical experiments is found to be very comparable to the spread in values contained between the Reuss and Voigt bounds for the polycrystal model. However, computed Hashin-Shtrikman bounds are much too tight to be in agreement with the numerical data, showing that polycrystals of cracked grains tend to violate some implicit assumptions of the Hashin-Shtrikman bounding approach. However, the self-consistent estimates obtained for the random polycrystal model are nevertheless very good estimators of the observed average behavior.
\end{abstract}

PACS numbers: 46.50.+a,61.72.Qq,62.20.-x,62.20.Mk,68.60.Bs,91.60.Ba

* JGBerryman@LBL . GOV 
†Vladimir.Grechka@Shell.Com 


\section{INTRODUCTION}

It is known $[1,2]$ that the quasistatic behavior of cracked or fractured systems cannot be successfully modeled using traditional effective medium theory methods: (a) in part because most of those methods are based on energy stored in the volume of the inclusions, whereas the effects of cracks are known to be nearly independent of their volume fraction, and (b) in part because addition of more cracks results in shielding effects, thereby reducing the softening influence of all the cracks on each other and on the overall system. This reduced softening effect for multiple cracks is usually missed entirely by the traditional volumefraction-based effective medium theories, typically based on Eshelby's analysis of ellipsoidal inclusions [3].

To circumvent the volume fraction issue, we consider herein a model based on grains containing cracks. The grains are assembled into (for example) an isotropic polycrystal of cracked grains. (The assumed overall isotropy is not a requirement of the approach, but it does greatly simplify presentation of the modeling results.) The analysis of polycrystal behavior then proceeds using ensemble averaging and, therefore, is not limited by the lack of crack-volume sensitivity of such cracked systems. This model of polycrystals of cracked grains also contains within it an effect similar to the shielding effect observed in high crackdensity systems. In particular, it is not difficult to show that the natural definition of the bulk modulus of an anisotropic grain is always given precisely by the Reuss average of the bulk modulus. (Imagine immersing a grain in a water bath, and then measuring total grain strain as a function of fluid pressure.) But since the same Reuss average is also the rigorous lower bound of the bulk modulus of a polycrystal composed of like grains, it is certain that a polycrystal of grains will be hydrostatically stiffer than the grains themselves [4]. We can attribute this effect to grain-to-grain bridging of the strongest components (i.e., the large volume of solid that is not cracked in the present study). The effect just described will always be present in true polycrystals, and may be contributing part of the observed "shielding" in cracked systems. But, we do not expect that this is the only type of crack-crack shielding present in real systems. In particular, the assumed granular structure of polycrystals also prevents various long-range connections among cracks from occurring, and thus limits the range of behaviors that can be present in the model; by assumption, cracks never intersect grain boundaries in these models, so these systems are thereby inherently constrained never 
to fail (either locally or globally) in the elastic regime to which the analysis is restricted.

We first provide an overview of the technical approach based on random polycrystals of cracked grains in Section II; the details of the method are contained in Appendix A. Then, two sets of numerical examples are analyzed using the polycrystal approach in Section III. To motivate the method used to fit the polycrystal analysis output to these numerical data, we also make use of the crack-influence decomposition method of Sayers and Kachanov [5]. The pertinent details of this method are presented in Appendix B. Our results and conclusions are discussed in the final section.

\section{MODELING APPROACH}

The technical aspects of the modeling approach follow the results contained in Ref. [6], and are summarized in Appendix A. The main idea is based on several facts about polycrystals and the technical issues associated with estimating their properties.

The first and most obvious issue with cracked materials is that, in the vicinity of one crack, the effective elastic properties will necessarily be anisotropic. Since by far the great majority of effective medium and other upscaling methods for composite materials have been designed for overall isotropic composites composed of isotropic constituents, we necessarily must try something rather different for cracked materials. It seems natural to suppose that the cracked medium may be viewed as a collection of randomly oriented grains of various sizes and orientations containing cracks. For the dilute case having rather small crack densities, we could imagine that each grain has only one crack per grain. But, as the crack density increases, cracks may intersect with other cracks. In this case, we may have multiple cracks and/or intersecting cracks in each grain.

The shape of the cracked grains is another possible variable within the model. It is fairly common in the study of polycrystals to assume that the grains are spherical [7]. Since it is clear that it is not possible to fill all of space with spheres of the same size, such a model requires a further assumption that the grains come in a wide variety of sizes down to the infinitesimal and that this happens in a way that does fill all space. It seems clear that this type of model is artificial in the sense that it applies to no real material, but it nevertheless is a model that has been used extensively in effective medium theories and generally seems to produce reasonable modeling results. Nevertheless, we will avoid this approach here and 
consider instead an alternative wherein we assume that the cracks come in clusters. A cluster might be just one crack, or several cracks in close proximity. Then we assume each cluster is sufficiently separated from the other crack clusters that we can assign a center point to each cluster and then construct Voronoi polyhedra by drawing planes midway between nearest neighbors so that all of space is partitioned without leaving any holes. Figure 1 illustrates one realization for this model random polycrystal of cracked grains.

Various rigorous bounds are known for polycrystals (Voigt, Reuss, Hashin-Shtrikman [810]), and the commonly used Voigt-Reuss-Hill estimate (arithmetic average of the Voigt and Reuss bounds [11]) is both well-known and well-established. It is important to note however that the Hashin-Shtrikman bounds are problematic for the polycrystalline case because it is known that for composites of anisotropic components the Voigt bound is achievable in certain special cases [12]. This fact is sufficient to invalidate the use of the Hashin-Shtrikman bounds as a general method (since the upper HS bound is always smaller than the Voigt bound, thus precluding the possibility of achieving the Voigt bound in the cases where this is known to be an exact result - IF the HS upper bound were valid). Thus, HS bounds must be used cautiously, if at all for anisotropic media, and with the understanding that there are various implicit assumptions in the HS theory that may or may not be satisfied in a given composite having anisotropic constituents [12].

On the other hand, there are also general self-consistent estimators available for these same anisotropic composites [6, 13, 14]. These self-consistent estimators actually do lie between the Hashin-Shtrikman bounds, but - since they are merely estimates of the average behavior and not bounds in any sense - they do not suffer from the same questions concerning their validity as the HS bounds themselves. These self-consistent estimates for polycrystals have approximately the same theoretical significance as the Voigt-Reuss-Hill estimates, and should therefore provide generally useful quantitative measures of expected average elastic behavior of polycrystals. We use both types of estimates (Voigt-Reuss-Hill and self-consistent) here when we need values of effective bulk and shear moduli: (1) we use the VRH approach as a means of estimating the effective bulk and shear moduli that can be associated with the stiffness matrices generated by the numerical methods and (2) we use the self-consistent estimator as a means of quantifying the average behavior associated with our predictions and/or fitted values for the polycrystal model.

In the following examples, we find that the HS bounds are in fact much too close together 
to be useful in explaining the range of behavior observed in the numerical data considered here. However, the Voigt and Reuss bounds do give very good estimates for the range in behavior, while the self-consistent estimator gives a good estimate of the average behavior.

\section{EXAMPLES}

Grechka and Kachanov [1,2] have generated two distinct numerical data sets for crack densities $\rho=0.05,0.10,0.15$, and 0.20 . (Crack density is defined as $\rho=n a^{3}$, where $n$ is the number density per unit volume. Radius of a typical crack is $a$. If there is a distribution of crack sizes, then $n a^{3}$ is replaced by an appropriate average value.) Cracks are all pennyshaped, but not necessarily flat. We assume that the aspect ratio is small (but finite, i.e., nonzero) and, therefore, we can ignore it here.

The two models considered have very different Poisson's ratios for the isotropic background media: (1) $\nu_{0}=0.00$ and (2) $\nu_{0}=0.4375$. We will call these two models, respectively, the first model and the second model. The first model has background stiffness matrix values $c_{11}=c_{22}=c_{33}=13.75 \mathrm{GPa}, c_{12}=c_{13}=c_{23}=0.00 \mathrm{GPa}$, and $c_{44}=c_{55}=c_{66}=6.875$ GPa. Bulk modulus for this model is therefore $K_{0}=4.583 \mathrm{GPa}$ and shear modulus is $G_{0}=6.875 \mathrm{GPa}$. The second model has stiffness matrix values $c_{11}=c_{22}=c_{33}=19.80 \mathrm{GPa}$, $c_{12}=c_{13}=c_{23}=15.40 \mathrm{GPa}$, and $c_{44}=c_{55}=c_{66}=2.20 \mathrm{GPa}$. Bulk modulus for this model is therefore $K_{0}=16.86 \mathrm{GPa}$ and shear modulus is $G_{0}=2.20 \mathrm{GPa}$. The second model also corresponds to a background material having compressional wave speed $V_{p}=3 \mathrm{~km} / \mathrm{s}$, shear wave speed $V_{s}=1 \mathrm{~km} / \mathrm{s}$, and mass density $\rho_{m}=2200.0 \mathrm{~kg} / \mathrm{m}^{3}$.

The numerical modeling of Grechka and Kachanov $[1,2,15]$ for the first model resulted in 110 examples: 23 for $\rho=0.05,21$ for $\rho=0.10,35$ for $\rho=0.15$, and 31 for $\rho=0.20$. Of

these 110 cases, we used 79 (selected randomly for the three smaller crack densities): 8 for $\rho=0.05,16$ for $\rho=0.10,24$ for $\rho=0.15$, and all 31 for $\rho=0.20$.

The numerical modeling of Grechka and Kachanov $[1,2,15]$ for the second model resulted in 100 examples: 25 each for all four cases $\rho=0.05,0.10,0.15,0.20$. Of these 100 cases, we used 73 (selected randomly for the three smaller crack densities): 8 for $\rho=0.05,16$ for $\rho=0.10,24$ for $\rho=0.15$, and all 25 for $\rho=0.20$.

For both numerical data sets, the output of the modeling for each example was a stiffness matrix. All these matrices were observed to be essentially orthotropic in character (i.e., 
depending significantly only on the nine stiffness constants $c_{11}, c_{22}, c_{33}, c_{12}=c_{21}, c_{13}=c_{31}$, $c_{23}=c_{32}, c_{44}, c_{55}, c_{66}$ - meaning that the remaining off-diagonal coefficients are negligible). In order to produce results useful for graphical comparisons, these orthotropic matrices were used to compute the Voigt-Reuss-Hill averages of bulk and shear moduli. So the Voigt and Reuss averages $K_{V}$ and $K_{R}$ were computed for each matrix, and arithmetic average $K_{V R H}=\left(K_{R}+K_{V}\right) / 2$ was obtained as a measure of the bulk modulus for the stiffness matrix. Then, a similar calculation was done for the shear modulus $G_{V R H}$. Formulas for all these averages are given for orthotropic elastic stiffness matrices by Watt [16]. The results $(\mathrm{VRH})$ are plotted as red $\times$ 's in Figures 2-9, where the numerical results are also compared to results from the differential scheme (DS), the non-interacting approximation (NI), and various bounds and estimates based on random polycrystals of cracked grains $\left(\mathrm{R}, \mathrm{HS}^{-}, \mathrm{SC}, \mathrm{HS}^{+}, \mathrm{V}\right)$.

We also show two other estimates: SMP for "sample," and GR for "grain." Since these computations were all done using displacement boundary conditions, we have made use of results from Huet [17] on sampling — the pertinent result being that effective overall stiffness $C_{i j}^{*}$ of a random medium (one having a well-defined statistical ensemble associated with it) satisfies $C_{i j}^{*} \leq C_{i j}^{S M P} \leq C_{i j}^{V}$, where

$$
C_{i j}^{S M P}=\frac{1}{N} \sum_{n=1}^{N} C_{i j}^{n}
$$

and where the $C_{i j}^{n}$ are subsamples of the collection of local stiffnesses in the ensemble. For present purposes, we can choose to think of the individual stiffness matrices found in the numerical experiments as being just such representatives selected randomly from the overall ensemble. Then, the SMP value we use is the one based on the values $N=8,16,24$, etc., for the cases considered in the numerical experiments. Clearly, the value we obtain this way for $C_{i j}^{S M P}$ is merely an estimate of the true average value, based on our rather limited statistics. But we find that the fluctuations in these averages are quite small already with the stated sample numbers used here, and so we conclude that the approach is in fact useful even for such apparently small sample statistics. Furthermore, the plotted values $K_{S M P}$ and $G_{S M P}$ are the Voigt averages for bulk and shear modulus obtained from the stiffness average $C_{i j}^{S M P}$. For comparison purposes, we also provide the values $K_{G R}$ and $G_{G R}$, which are the Reuss averages associated again with stiffness average $C_{i j}^{S M P}$. These values have no special theoretical significance in terms of the analysis of Huet [17], as the more appropriate bounding 
values from below should be obtained - not from the stiffness obtained for displacement boundary conditions but - instead for the compliance determined in a numerical experiment applying traction boundary conditions. But for the available data sets, we did not have this additional information. One further motivation, however, for presenting these values $K_{G R}$ and $G_{G R}$ is that the stiffness $C_{i j}^{S M P}$ is in some fairly precise sense the stiffness of an "average" cracked grain in the overall polycrystal model. The bulk modulus of this "average" cracked grain is given precisely by the value $K_{G R}$, but there is no corresponding statement that can be made about the shear modulus of this same average grain. So $G_{G R}$ is just a special estimate, or heuristic value, that can be easily computed, and its significance should therefore not be overinterpreted.

One class of approximations that has been found very useful for analysis of these cracked systems by Grechka and Kachanov [1,2] is the non-interaction approximation (NIA). Since our results will be presented in the form of plots of effective bulk and shear moduli, it is useful to consider the corresponding NIA formulas for bulk and shear moduli. Zimmerman [18] gives such formulas, and in our present notation these formulas are:

$$
\frac{K_{0}}{K_{N I}}=1+\rho \frac{16\left(1-\nu_{0}^{2}\right)}{9\left(1-2 \nu_{0}\right)}
$$

for effective bulk modulus $K_{N I}$, and

$$
\frac{G_{0}}{G_{N I}}=1+\rho \frac{32\left(1-\nu_{0}\right)\left(5-\nu_{0}\right)}{45\left(2-\nu_{0}\right)}
$$

for effective shear modulus $G_{N I}$. The host medium has bulk modulus $K_{0}$, shear modulus $G_{0}$, and Poisson's ratio $\nu_{0}=\left(3 K_{0}-2 G_{0}\right) / 2\left(3 K_{0}+G_{0}\right)$. Again, the crack density $\rho=n a^{3}$, where $n$ is the number of cracks per unity volume, and $a$ is the radius of the (assumed) penny-shaped cracks. In particular, we note that in a plot of inverse bulk modulus and/or inverse shear modulus versus crack density $\rho$, the NIA results are just straight lines. This general feature of NIA suggests that it is most fruitful to construct our plots in this way in order to distinguish easily whether the results are behaving according to NIA predictions - or not. In fact, we find that, although the NIA gives good agreement for some of the numerical results, in general there are deviations from NIA, and that the polycrystal of cracked grains model gives a better representation of the numerical results.

Another method that we use here involves a crack-influence decomposition method of Sayers and Kachanov [5]; see Appendix B for details. When all cracks in the system have 
the same vertical ( $z$-)axis of symmetry, then the cracked/fractured system is not isotropic, and we have the compliance correction matrix

$$
\Delta S_{i j}=\rho\left(\begin{array}{ccccc}
0 & 0 & \eta_{1} & & \\
0 & 0 & \eta_{1} & & \\
\eta_{1} & \eta_{1} & 2\left(\eta_{1}+\eta_{2}\right) & & \\
& & & & \\
& & & & \\
& & & & \\
& & & & \\
& & & & 0
\end{array}\right)
$$

(In fact, we will show later that $\eta_{1}$ corrections in (4) are usually negligible compared to the $\eta_{2}$ corrections. This also holds true in other formulas for compliance corrections, but we nevertheless carry $\eta_{1}$ along in the formulas for completeness [see (15)].) Now it is also not difficult to see that, if the cracks were oriented instead so that all their normals were pointed horizontally along the $x$-axis, then we would have one permutation of this matrix and, if instead they were all pointed horizontally along the $y$-axis, then we would have a third permutation of the matrix. Averaging these three permutations by adding the three $\Delta S$ 's together and then dividing by three, we obtain the isotropic compliance corrections matrix

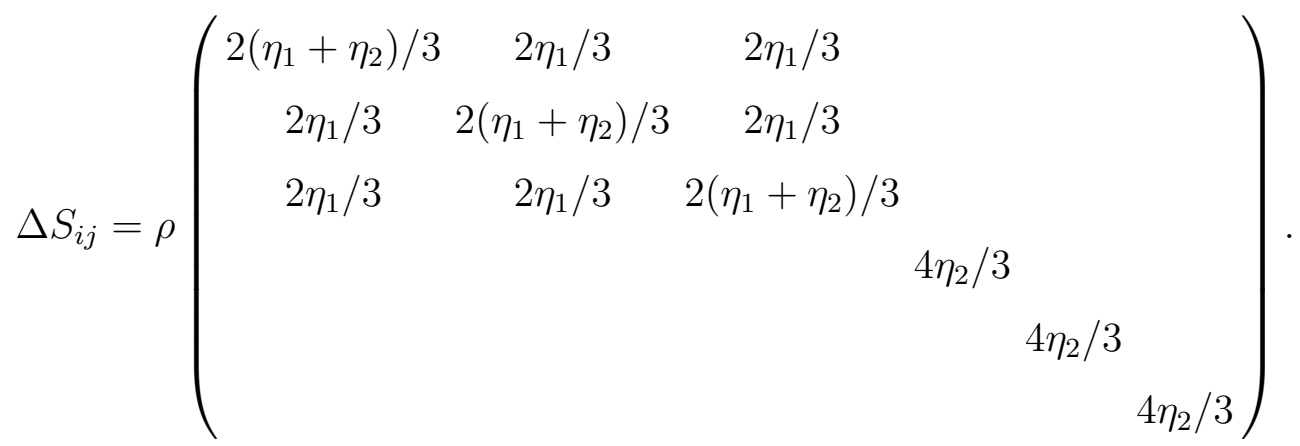

Then, since the unperturbed compliance matrix is related to Young's modulus $E_{0}$, Poisson's ratio $\nu_{0}$, and shear modulus $G_{0}$ by

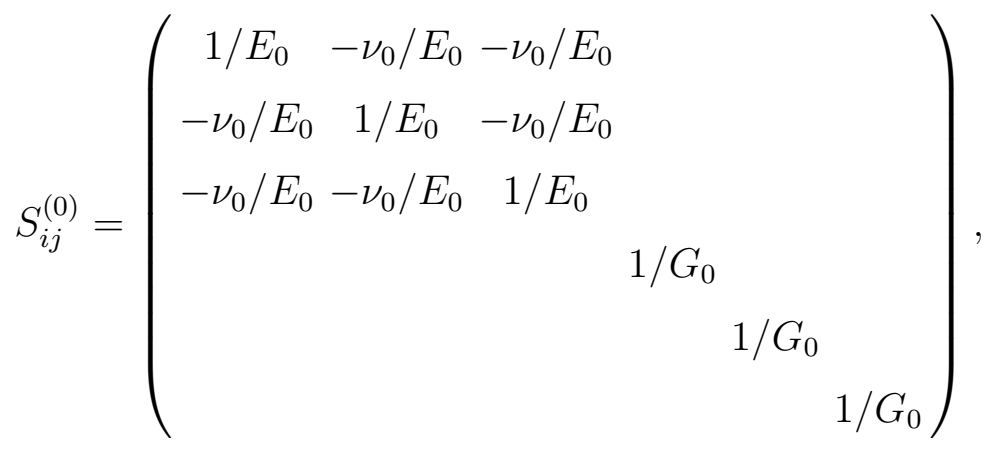


we find easily that

$$
\frac{1}{E^{*}}=\frac{1}{E_{0}}+\frac{2}{3} \rho\left(\eta_{1}+\eta_{2}\right)
$$

and

$$
\frac{-\nu^{*}}{E^{*}}=\frac{-\nu_{0}}{E_{0}}+\frac{2}{3} \rho \eta_{1}
$$

Solving these equations for the $\eta$ 's, we have

$$
\eta_{1}=-\frac{3}{2 \rho}\left(\frac{\nu^{*}}{E^{*}}-\frac{\nu_{0}}{E_{0}}\right)
$$

and

$$
\eta_{2}=\frac{3}{2 \rho}\left(\frac{\left(1+\nu^{*}\right)}{E^{*}}-\frac{\left(1+\nu_{0}\right)}{E_{0}}\right) .
$$

Since $1 / G_{0}=2\left(1+\nu_{0}\right) / E_{0}$ for an isotropic system, we also have the consistency check that

$$
\eta_{2}=\frac{3}{4 \rho}\left(\frac{1}{G^{*}}-\frac{1}{G_{0}}\right)
$$

Similarly, since $1 / K_{0}=3\left(1-2 \nu_{0}\right) / E_{0}$, we also have

$$
\frac{1}{K^{*}}-\frac{1}{K_{0}}=2 \rho\left(\eta_{2}+3 \eta_{1}\right)
$$

This construction shows in part both the power and the simplicity of the Sayers and Kachanov [5, 19] approach.

The crack influence decomposition parameters $\eta_{1}$ and $\eta_{2}$ can be evaluated using the formulas presented for any convenient value of the crack density $\rho$. But this procedure is seen to be most useful if we evaluate the parameters at small $\rho$, since in that limit all the standard methods should give essentially the same results. This approach has been tested and found to be correct.

Once it is known that it makes little difference at low crack densities which theoretical methods we use to estimate the crack-influence parameters, we might as well consider the simplest one, which is surely the non-interaction approximation. Combining (2) and (3) with (11) and (12), we find easily that, within the NIA,

$$
\eta_{2}=\frac{8\left(1-\nu_{0}\right)\left(5-\nu_{0}\right)}{15 G_{0}\left(2-\nu_{0}\right)},
$$

and

$$
\eta_{1}=-\frac{4 \nu_{0}\left(1-\nu_{0}\right)}{15 G_{0}\left(2-\nu_{0}\right)}
$$


The ratio of these expressions is

$$
\eta_{1} / \eta_{2}=-\frac{\nu_{0}}{2\left(5-\nu_{0}\right)}
$$

This shows that, when $0 \leq \nu_{0} \leq 0.5$,

$$
\left|\eta_{1} / \eta_{2}\right| \leq 0.05
$$

So, $\left|\eta_{1}\right|$ is never larger than about $5 \%$ of $\eta_{2}$, and, for small values of $\nu_{0}$, the ratio is substantially smaller. A typical value for rocks, and especially for porous rocks, is $\nu_{0} \simeq 0.2$. It follows that the value of $\left|\eta_{1}\right|$ is about $2 \%$ of that for $\eta_{2}$ in geophysical applications. Thus, its value is suffficiently small so that we are often justified in neglecting $\eta_{1}$ in data analysis problems for real earth systems and rocks.

Once values of $\eta_{1}(0)$ and $\eta_{2}(0)$ are known in this way (using NIA as shown or some other method), we can use the Sayers and Kachanov method [5] as one convenient way to study and evaluate anisotropic behavior in cracked systems - thus, providing a simple method of extending the non-interaction approximation results, as quoted by Zimmerman [18], to nonisotropic systems. We then introduce some crack-crack interactions here in a novel way by making use of the polycrystal of cracked-grains model.

Appendix B summarizes the main analysis using the crack-influence parameter approach of the Sayers and Kachanov method [5]. We find that, in addition to the two parameters $\eta_{1}$ and $\eta_{2}$ that are easily found using effective medium theories such as NIA or DS (differential scheme), there are three more parameters that are expected to play a role in our results at higher crack densities: $\eta_{3}, \eta_{4}$, and $\eta_{5}$. These are the only significant quadratic corrections to (4). The analysis shows [see, for example, Eq. (40)] that to second order in the crack density $\rho$, we need to replace $\eta_{1}$ by $\eta_{1}+\eta_{4} \rho$ and $\eta_{2}$ by $\eta_{2}+\eta_{5} \rho$. In addition, the parameter $\eta_{3}$ comes into play, but perturbs only the $S_{33}$ component of the compliance. The Reuss averages of shear modulus and bulk modulus are now given by

$$
\frac{1}{G_{R}}-\frac{1}{G_{0}}=\frac{4 \rho}{3}\left[\eta_{2}+\left(\eta_{5}+2 \eta_{3} / 5\right) \rho\right]
$$

and

$$
\frac{1}{K_{R}}-\frac{1}{K_{0}}=2 \rho\left[\eta_{2}+\left(\eta_{3}+\eta_{5}\right) \rho+3\left(\eta_{1}+\eta_{4} \rho\right)\right]
$$

respectively, instead of (11) and (12). Furthermore, since it has been found empirically that the shear modulus estimates given by the NIA are quite accurate for the polycrystals 
of cracked-grains model, we conclude that it is generally true that $\eta_{5}+2 \eta_{3} / 5 \simeq 0$, which approximately eliminates one degree of freedom in our three parameter $\left(\eta_{3}, \eta_{4}, \eta_{5}\right)$ fitting method.

Also, recall that we are focusing here on penny-shaped (and therefore ellipsoidal) cracks. Phenomenology for other crack shapes may differ somewhat from the discussion presented here [20]. However, other shapes are beyond our present scope and will necessarily be treated elsewhere. The numerical experiments considered here all used ellipsoidal cracks.

\section{A. Results for first model}

Results of both models will be presented in two distinct sets. The first set involves no fitting of the data, while the second set does involve data fitting.

The non-interaction approximation is particularly simple for the first model since $\nu_{0}=0.0$. Then, (3) and (2) show that

$$
\frac{G_{0}}{G_{N I}}=\frac{K_{0}}{K_{N I}}=1+\rho \frac{16}{9}
$$

and, therefore, we also have $\nu_{N I}=0.0$ for all $\rho$ in this approximation - since the proportionality between the bulk and shear moduli never changes for this case. Background bulk modulus for this model is $K_{0}=4.583 \mathrm{GPa}$ and corresponding shear modulus is $G_{0}=6.875$ GPa. In the Sayers and Kachanov scheme, $\eta_{1}(0)=0.0 \mathrm{GPa}^{-1}$ and $\eta_{2}(0)=0.1941 \mathrm{GPa}^{-1}$. All off-diagonal perturbations of the compliance matrix comes from $\eta_{1}$, so there is no change to the zero values off the diagonal for this case. For a single crack per grain and crack density $\rho$, all perturbations are of the form $\Delta=2 \rho \eta_{2}(0)$ and these perturbations are contributing

only to these three compliance values: $S_{33}, S_{44}$ and $S_{55}$. The compliance matrix may next be inverted to produce the perturbed stiffness matrix, and then these values are used in the formulas in Appendix A and also in the Figures.

\section{Without fitting}

Figures 2 and 3 show the results for the first model using only the results of the NIA as input to the polycrystal of cracked-grains model. For comparison, we also display the NIA results, the differential scheme (DS) effective medium theory results [18, 21], and the numerical data $(\mathrm{x})$. The numerical data were actually stiffness matrices, so these matrices 
have been converted to Voigt-Reuss-Hill (VRH) estimates of shear and bulk moduli for plotting and comparison purposes. All curves converge at low crack densities as they should. We see that the numerical data deviate from the NIA curve substantially for both shear and bulk moduli. But the deviations are especially strong for the bulk modulus estimates. Without fitting, the shear modulus estimates have values that are about equal to the selfconsistent polycrystal estimates, or higher (note the plots are inverse moduli). In contrast, the bulk modulus (inverse modulus) estimates are always significantly higher (lower) than the self-consistent estimates. We interpret this difference between the shear modulus and bulk modulus results as being due to the presence of longer range interactions for bulk modulus

effects, and shorter range interactions for shear modulus effects. Thus, even without any attempt at fitting, the polycrystal grain model appears to be a fairly good model for the shear behavior, but not as good for the bulk (hydrostatic) behavior.

We can modify the results for the polycrystals of cracked-grains model by including higher order corrections from the Sayers and Kachanov [5] model, as in the discussion accompanying Eqs. (38)-(41), and that is what we do next.

\section{With fitting}

When developing our approach to fitting the numerical the data, we took the basic model of Sayers and Kachanov [5], including the higher order corrections in powers of crack density, as discussed in Appendix B, and then tried to find the simplest set of coefficients that would fit the numerical data. Since the fit was already fairly good for shear modulus of the first model, and since it was mostly the bulk modulus that deviated very much from the numerical data, we determined that a method producing as little change as possible in the shear modulus, while still affecting the needed changes in bulk modulus, was what was needed.

Since $\eta_{1}=0.0$ for this case, $\eta_{4}=0.0$ also seemed the logical choice for this parameter. This leaves us with two crack-influence parameters to fit: $\eta_{3}$ and $\eta_{5}$. One type of increment that produces relatively small changes in shear modulus, while also changing bulk modulus, is one of the form $\Delta_{33}=2\left(\eta_{3}+\eta_{5}\right) \rho^{2}$ added to $S_{33}$, while at the same time adding corrections $\Delta_{44}=\Delta_{55}=-2 \Delta_{33} / 3$ to $S_{44}$ and $S_{55}$. This type of shift causes no change in the Reuss average for shear modulus. (In fact, we actually used this approach for the quadratic correc- 
tions in the second model, as will be seen in the subsequent discussion.) Although we tried this approach here, it did not seem to be as successful as desired at improving the fit to the numerical data. In particular, since the shear modulus agreement in this case could stand some improvement anyway, we modified this approach slightly and found (after some trial and error) that setting $\Delta_{44}=\Delta_{55}=2 \eta_{5} \rho^{2}=-\Delta_{33} / 3$ worked better in this case. This choice does not leave the Reuss average of shear modulus unchanged, but that was not an absolute condition we needed or wanted to impose here. By choosing $\Delta_{33}=-0.00275(\rho / 0.05)^{2} / 2$, we thus obtained the agreement seen in Figures 4 and 5, and no further searching for better fits to the data were pursued. These corrections are translated into numerical values for $\eta_{3}$ and $\eta_{5}$ in TABLE 1.

\section{B. Results for second model}

The non-interaction approximation is not quite so simple for the second model as it was for the first. In particular, having $\nu_{0}=0.4375$ for this model, we find that the NIA already gives more complicated behavior since

$$
\frac{K_{0}}{K_{N I}}=1+11.5 \rho
$$

for effective bulk modulus $K_{N I}$, and

$$
\frac{G_{0}}{G_{N I}}=1+1.168 \rho
$$

for effective shear modulus $G_{N I}$ where $K_{0}=16.86 \mathrm{GPa}, G_{0}=2.20 \mathrm{GPa}$, and $\nu_{0}=0.4375$. So Poisson's ratio does not remain constant for this NIA model.

In the Sayers and Kachanov scheme, $\eta_{1}(0)=-0.01920 \mathrm{GPa}^{-1}$ and $\eta_{2}(0)=0.3994 \mathrm{GPa}^{-1}$, as determined by the differential scheme (DS).

\section{Without fitting}

Results for the second model using only the NIA as input to the random polycrystal of cracked-grains model are displayed in Figures 6 and 7. Again, for comparison, we also show the outputs from the same set of theoretical approaches as before. Stiffness matrix data were again converted to Voigt-Reuss-Hill estimates of shear and bulk moduli for the 
plots. All curves converge at low crack densities, and we find the numerical data deviate from the NIA curve substantially for both shear and bulk moduli. But deviations of the numerical data from the random polycrystal method predictions are especially strong for the bulk modulus estimates. With no fitting, the shear modulus estimates have values that are centered about the self-consistent polycrystal estimates, and that lie between the Voigt and Reuss bounds. This shear modulus agreement (but without fitting) is actually better than that of the corresponding case for the first model. In contrast, the bulk modulus estimates are always significantly higher than even the Voigt upper bound (recall that the plots are inverse moduli). Again we interpret this difference between shear modulus and bulk modulus results as probably being due to the presence of longer range interactions for bulk modulus effects, and shorter range interactions for shear modulus effects. Also, the random polycrystal grain model appears to be a good model for the shear behavior, but in this case a considerably worse model for the bulk behavior — at least until the quadratic corrections are applied.

Again, we can modify the results of the polycrystals of cracked grains model by including higher order corrections from the Sayers and Kachanov [5] model, and do so now.

\section{With fitting}

Fitting the numerical data using linear, as well as quadratic, corrections to the basic Sayers and Kachanov [5] approach, we obtain Figures 8 and 9. This second model is also more complicated than the first since it requires us to consider both diagonal and off-diagonal contributions to the compliance matrix. In particular, Figure 8 shows the bulk modulus is greatly underpredicted. But this time it is both possible and desirable to make off-diagonal corrections. The problem in doing so is that the initial shear modulus estimates are already so good that it would be preferable to make changes that do not affect the quality of the shear modulus results - assuming that this is possible. One type of change that yields the desired behavior is a uniform change to all the coefficients having to do with the principal stresses and strains. So we might want to make changes only to $S_{11}, S_{22}, S_{33}, S_{12}=S_{21}$, $S_{13}=S_{31}$, and $S_{23}=S_{32}$. Changing them all by the same amount will not affect the Reuss average for shear modulus, but may affect the Voigt average and the self-consistent estimate. However, we found again that $\eta_{4}=0.0$ was an appropriate value, and therefore did not make 
any change of this type.

Another alternative discussed previously that produces relatively small changes in shear modulus, while also changing bulk modulus, is one of the form $\Delta_{33}=2\left(\eta_{3}+\eta_{5}\right) \rho^{2}$ added to $S_{33}$, while at the same time adding corrections $\Delta_{44}=\Delta_{55}=2 \eta_{5} \rho^{2}=-2 \Delta_{33} / 3$ to $S_{44}$ and $S_{55}$. This type of shift causes a very small or no change in the Reuss average for shear. By choosing $\Delta_{33}=-0.00825(\rho / 0.05)^{2} / 2$, we obtained the results observed in Figures 8 and 9. The numerical values were chosen by trial and error, based on the results observed in the plots. We chose to fit the values at the highest available values of crack density, even though this seemed to force the fit at lower crack densities to be worse than could have been achieved with other parameter values. We do not claim that our search has been exhaustive. There might be better choices to be made, and especially so if the number of $\eta$ parameters included in the search were increased. The observed fit is certainly not as good for this second model as it was for the first.

Final results for the significant crack-influence parameters of both models are summarized in TABLE 1.

TABLE 1. Values of five crack-influence parameters for the two models considered.

\begin{tabular}{|c|c|c|}
\hline \hline Crack-influence & First Model & Second Model \\
Parameters & $\nu_{0}=0.00$ & $\nu_{0}=0.4375$ \\
\hline$\eta_{1}(0)\left(\mathrm{GPa}^{-1}\right)$ & 0.0000 & -0.0192 \\
$\eta_{2}(0)\left(\mathrm{GPa}^{-1}\right)$ & 0.1941 & 0.3994 \\
$\eta_{3}(0)\left(\mathrm{GPa}^{-1}\right)$ & -0.3666 & -1.3750 \\
$\eta_{4}(0)\left(\mathrm{GPa}^{-1}\right)$ & 0.0000 & 0.0000 \\
$\eta_{5}(0)\left(\mathrm{GPa}^{-1}\right)$ & 0.0917 & 0.5500 \\
\hline \hline
\end{tabular}

\section{DISCUSSION AND CONCLUSIONS}

Standard effective medium theories (differential scheme, self-consistent scheme, etc.), when applied to fractured systems, all overpredict the influence of the cracks. Of all the standard effective medium theories, the best one from this point of view is clearly the noninteraction approximation (NIA) because it assumes there is no influence of one crack on 
any other, while — with this one exception — all the other effective medium theories predict that multiple cracks enhance the effects of each other. But numerical experiments show that the actual behavior is the opposite; when cracks interact, they screen or shield each other and reduce the overall effects of the cracks that are present. So NIA is best one within this restricted class of methods in the sense that, unlike all the others, it does not go in the wrong direction at higher crack densities.

The main question then becomes whether or not there is some simple theoretical method that gives the right correction, i.e., introduces crack shielding and, therefore, stiffer (less compliant) results than all these other methods.

This question has been answered in the affirmative in this paper by introducing and studying the quasistatic behavior of the random polycrystals of cracked-grains model. Each grain has one or more cracks and is therefore anisotropic, having either hexagonal or perhaps more generally orthotropic symmetry. But each of these grains will tend to be weakest in the direction normal to its main crack, and strongest in the two orthogonal directions defined by the plane of the main crack (or cracks). So, when these cracked grains are jumbled together into an isotropic composite medium, the overall effect includes a high proportion (2 to 1 ratio) of strong directions interacting with the strong directions of other grains. This interaction ultimately produces bridging across the entire composite material and is a guaranteed result for bulk modulus (hydrostatic) behavior since the bulk modulus of each grain is determined exactly by its Reuss average (and therefore the weakest possible combination of elastic constants), while the bulk behavior of the composite polycrystal is dominated instead by the strongest connected paths passing throughout the whole composite. From this point of view, for small to moderate values of crack density, we see that fractures/cracks exist as isolated weak patches in an otherwise comparatively strong background framing material. When fractures/cracks intersect only locally, this basic picture does not change qualitatively. But clearly if the crack density increases to the point where cracks start coalescing and spanning the entire composite, then their effect is multiplied and the composite medium can become fragmented thus basically having no strength overall under such circumstances. We have assumed here that all the cases we were studying are far from this extreme limit of the theory, and of course that is also consistent with the types of numerical data used in our comparisons with the theory.

We conclude that the random polycrystals of cracked-grains model is a useful approach to 
the modeling of small to moderate crack density (i.e., $0<\rho \leq 0.1$ ) materials. The method involves established formulas from the theory of composites including both bounds and self-consistent estimates for polycrystals. This approach has apparently not been applied to this class of problems previously. Results for shear modulus prediction were found to be especially good, as the simplest method tried actually worked quite well in both of the numerical modeling experiments considered here. Getting the theory to agree simultaneously with the bulk and shear behavior required the introduction of some quadratic corrections, whose coefficients were treated as fitting parameters. This approach is consistent with the crack-influence decomposition method of Sayers and Kachanov [5]. One quadratic fitting parameter was sufficient to produce a good fit when Poisson's ratio $\nu_{0}=0.0$. Only one parameter was also used at first for the case $\nu_{0}=0.4375$. But the results were not an unqualified success in this case. Actual fitting to the numerical data was clearly better for the first model, but fitting was surely adequate for most practical purposes in both cases. Clearly the basic trends have been captured using this approach, involving only one fitting parameter.

In the cases considered, the Hashin-Shtrikman bounds were always too close together to give any useful estimate of the range of scatter in the numerical data for the cracked media. (This fact shows that the assumptions implicit in that derivation of the HS bounds have been violated in the random crack problem.) On the other hand, the differences between Voigt and Reuss bounds gave reasonably accurate estimates of the range of values seen in the numerical data. In one example, the actual highest and lowest values for both bulk and shear modulus were also well-constrained. These results depend to some extent on choosing appropriate fitting parameters for the corrections quadratic in crack density. The self-consistent estimate for the polycrystal analysis lies approximately in the middle of the region bounded by the Voigt and Reuss bounds, and so seems again to be a useful estimate - subject however to the same caveats (of fitting) as for the Voigt and Reuss bounds. A more satisfying result would have been establishing some means of predicting the values of these coefficients (i.e., the $\eta$ 's) of the quadratic fitting terms, but doing so in a meaningful way will require more numerical data than is available at the present time and/or some new theoretical analysis of this problem.

This study was clearly limited in its potential scope by the quantity of numerical data available. In particular, it would have been helpful to have some intermediate values of 
Poisson's ratio $0.0<\nu_{0}<0.4375$, so that the dependence of the quadratic fitting parameters on Poisson's ratio could be determined. With just two values of $\nu_{0}$, this goal could not be achieved here as the simplest possible fit (and really the only fit possible with just two data points) is clearly a straight line - but this fact by itself does not contain any useful information. Work currently in progress will address these, as well as other related, issues concerning quality of fit, and number of parameters needed to achieve good fits across a greater selection of Poisson's ratios.

\section{ACKNOWLEDGMENTS}

JGB thanks Gareth Block, Mark Kachanov, Ernest L. Majer, and Joseph P. Morris for helpful discussions and comments on the manuscript. Work of JGB performed under the auspices of the U.S. Department of Energy, first at the University of California, Lawrence Livermore National Laboratory under Contract No. W-7405-ENG-48, and subsequently at the Lawrence Berkeley National Laboratory under Contract No. DE-AC03-76SF00098. Support at both institutions was provided specifically by the Geosciences Research Program of the DOE Office of Basic Energy Sciences, Division of Chemical Sciences, Geosciences and Biosciences. VG thanks Shell E \& P, Inc. for supporting the modeling effort.

[1] V. Grechka and M. Kachanov, Geophysics 71, D85 (2006).

[2] V. Grechka and M. Kachanov, Geophysics 71, D93 (2006).

[3] J. D. Eshelby, Proc. Royal Soc. London A 241, 376 (1957).

[4] J. G. Berryman, J. Appl. Phys. 96, 4281 (2004).

[5] C. M. Sayers and M. Kachanov, Int. J. Solids Struct. 27, 671 (1991).

[6] J. G. Berryman, J. Mech. Phys. Solids 53, 2141 (2005).

[7] T. Olson and M. Avellaneda, J. Appl. Phys. 71, 4455 (1992).

[8] W. Voigt, Lehrbuch der Kristallphysik (Teubner, Leipzig, 1928).

[9] A. Reuss, Z. Angew. Math. Mech. 9, 49 (1929).

[10] Z. Hashin and S. Shtrikman, J. Mech. Phys. Solids 10, 343 (1962).

[11] R. Hill, Proc. Phys. Soc. London A 65, 349 (1952). 
[12] G. W. Milton, The Theory of Composites (Cambridge University Press, Cambridge, UK, 2002).

[13] J. R. Willis, J. Mech. Phys. Solids 25, 185 (1977).

[14] J. R. Willis, in Advances in Applied Mechanics, edited by C.-S. Yih (Academic Press, New York, 1981), vol. 21, pp. 1-78.

[15] V. Grechka, Stud. Geophys. Geod. 49, 365 (2005).

[16] J. P. Watt, J. Appl. Phys. 50, 6290 (1979).

[17] C. Huet, J. Mech. Phys. Solids 38, 813 (1990).

[18] R. W. Zimmerman, Compressibility of Sandstones (Elsevier, Amsterdam, 1991).

[19] Z. P. Bažant and J. Planas, Fracture and Size Effect in Concrete and Other Quasibrittle Materials (CRC Press, Boca Raton, Louisiana, 1998).

[20] G. Mavko and A. Nur, J. Geophys. Res. 83, 4459 (1978).

[21] J. G. Berryman, S. R. Pride, and H. F. Wang, Geophys. J. Int. 151, 597 (2002).

[22] J. G. Berryman, Geophys. J. Int. 127, 415 (2004).

[23] Z. Hashin and S. Shtrikman, J. Mech. Phys. Solids 10, 335 (1962).

[24] Z. Hashin and S. Shtrikman, J. Mech. Phys. Solids 11, 127 (1963).

[25] L. Peselnick and R. Meister, J. Appl. Phys. 36, 2879 (1965).

[26] J. P. Watt and L. Peselnick, J. Appl. Phys. 51, 1525 (1980).

[27] M. Kachanov, ASCE J. Engineering Mech. 106, 1039 (1980).

[28] M. Kachanov and I. Sevostianov, Int. J. Solids and Structures 42, 309 (2005).

[29] W. M. Bruner, J. Geophys. Res. 81, 2573 (1976).

[30] F. S. Henyey and N. Pomphrey, Geophys. Res. Lett. 9, 903 (1982).

[31] A. N. Norris, Mech. Materials 4, 1 (1985).

[32] R. W. Zimmerman, Int. J. Rock Mech. Mining Sci. 21, 339 (1984).

[33] M. Avellaneda, Commun. Pure Appl. Math. 40, 527 (1987).

[34] R. J. O'Connell and B. Budiansky, J. Geophys. Res. 79, 5412 (1974).

[35] J. G. Berryman, J. Acoust. Soc. Am. 68, 1820 (1980).

[36] G. W. Milton, Communications Math. Phys. 99, 463 (1985).

[37] Y. Benveniste, Mech. Materials 6, 147 (1987).

[38] G. T. Kuster and M. Toksöz, Geophysics 39, 587 (1974).

[39] J. G. Berryman and P. A. Berge, Mech. Materials 22, 149 (1996). 


\section{Appendix A: Bounds and estimates for random polycrystals of grains having known}

symmetry

Although the numerical results for stiffness matrices of randomly cracked systems in the experiments of Grechka and Kachanov [1, 2] generally show orthorhombic symmetry, in fact the symmetry has also been observed to be very close to hexagonal in all cases. The hexagonal symmetry is especially pertinent for these numerical experiments when the averaging approach of Huet [17] is used as in (1) where it was found that the results obtained from sampling and then averaging these numerical stiffness matrices tend to be very nearly

hexagonal stiffnesses. For the present purposes, after averaging to obtain $C_{i j}^{S M P}$ according to (1), the next step has been to find the closest hexagonal stiffness matrix for these averaged matrices by using a least-squares fitting method. The resulting hexagonal matrix is the one used to compute $K_{S M P}$ and $G_{S M P}$ in the examples. These results were also used in the fitting methods to obtain various bounds and estimates when introducing quadratic corrections to the very low crack density results.

Also, note that the step taken to reduce the matrices to hexagonal symmetry was not necessary for computing the VRH estimates for the individual stiffness matrices originally obtained from the numerical experiments. $K_{V R H}$ and $G_{V R H}$ can both be computed easily for these stiffnesses using the formulas given by Watt [16] for orthorhomic symmetry. But, after the averaged matrices $C_{i j}^{S M P}$ were computed as in (1), these matrices were slightly "smoothed" (in addition to the averaging) so the results had exactly hexagonal symmetry. The least-squares fitting method producing these smoothed hexagonal results typically amounted to a change by just a single unit in the third significant digit of only one of the nine distinct — for orthotropy — matrix components.

Figure 1 illustrates the type of geometry envisioned for the random polycrystals of cracked-grains model.

\section{Voigt and Reuss Bounds for Hexagonal Symmetry}

For hexagonal symmetry, the nonzero stiffness constants are: $C_{11}, C_{12}, C_{13}=C_{23}, C_{33}$, $C_{44}=C_{55}$, and $C_{66}=\left(C_{11}-C_{12}\right) / 2$. We assume a vertical (i.e., 3 or z) axis of symmetry. In cases where this was not true of the numerical data, we permuted the axis definitions until 
it was true.

The Voigt average for bulk modulus of these hexagonal systems is well-known to be

$$
K_{V}=\left[2\left(C_{11}+C_{12}\right)+4 C_{13}+C_{33}\right] / 9
$$

Similarly, for the shear modulus we have

$$
G_{V}=\frac{1}{5}\left(G_{\mathrm{eff}}^{v}+2 C_{44}+2 C_{66}\right)
$$

where the new term appearing here is essentially defined by (23) and given explicitly by

$$
G_{\mathrm{eff}}^{v}=\left(C_{11}+C_{33}-2 C_{13}-C_{66}\right) / 3
$$

The quantity $G_{\text {eff }}^{v}$ is the energy per unit volume in a grain when a pure "uniaxial shear" strain of unit magnitude $\left[i . e .,\left(e_{11}, e_{22}, e_{33}\right)=(1,1,-2) / \sqrt{6}\right]$, whose main compressive strain is applied to the grain along its axis of symmetry $[4,22]$.

The Reuss average for bulk modulus is determined by $1 / K_{R}=2\left(S_{11}+S_{12}\right)+4 S_{13}+S_{33}$, which can also be written as

$$
\frac{1}{K_{R}-C_{13}}=\frac{1}{C_{11}-C_{66}-C_{13}}+\frac{1}{C_{33}-C_{13}}
$$

in terms of stiffness coefficients. The Reuss average for shear is

$$
G_{R}=\left[\frac{1}{5}\left(\frac{1}{G_{\mathrm{eff}}^{r}}+\frac{2}{C_{44}}+\frac{2}{C_{66}}\right)\right]^{-1},
$$

which again may be taken as the definition of $G_{\mathrm{eff}}^{r}-i . e$. , the energy per unit volume in a grain when a pure uniaxial shear stress of unit magnitude $\left[\right.$ i.e., $\left.\left(\sigma_{11}, \sigma_{22}, \sigma_{33}\right)=(1,1,-2) / \sqrt{6}\right]$, whose main compressive pressure is applied to a grain along its axis of symmetry.

We use the following product formula as the formal definition of $G_{\text {eff }}^{r}$. For each grain having hexagonal symmetry, two product formulas hold [22]: $3 K_{R} G_{\text {eff }}^{v}=3 K_{V} G_{\text {eff }}^{r}=\omega_{+} \omega_{-} / 2=$ $C_{33}\left(C_{11}-C_{66}\right)-C_{13}^{2}$. The symbols $\omega_{ \pm}$stand for the quasi-compressional and quasi-uniaxialshear eigenvalues for the crystalline grains. Thus, $G_{\text {eff }}^{r}=K_{R} G_{\text {eff }}^{v} / K_{V}$ is a general formula that holds for all crystals having hexagonal symmetry. We can also treat (23) and (26) as the fundamental defining equations for $G_{\mathrm{eff}}^{v}$ and $G_{\mathrm{eff}}^{r}$, respectively.

\section{Peselnick-Meister-Watt (PMW) Bounds for Hexagonal Symmetry}

Hashin-Shtrikman-style bounds $[23,24]$ on the bulk and shear moduli of isotropic random polycrystals composed of grains having hexagonal symmetry have been derived by Peselnick 
and Meister [25], with corrections made later by Watt and Peselnick [26]. We will term these the PMW (for Peselnick-Meister-Watt) or the HS (Hashin-Shtrikman) bounds interchangably. The PMW notation was similar to that in the original Hashin-Shtrikman paper on random polycrystals of grains having cubic symmetry [10]. We will use a slightly modified notation here, taking into account the product formulas [22] in order to simplify the statement of the results. Derivations are found in the references, and therefore not repeated here.

Parameters used to optimize the Hashin-Shtrikman bounds are $K_{ \pm}$and $G_{ \pm}$, which have the significance of being the bulk and shear moduli of two $( \pm)$ isotropic comparison materials. $G_{+}, K_{+}$are the values used in the formulas for the upper bounds, and $G_{-}, K_{-}$for the lower bounds. Simplified formulas for the bulk modulus bounds are:

$$
K_{P M W}^{ \pm} \equiv K_{H S}^{ \pm}=\frac{K_{V}\left(G_{\mathrm{eff}}^{r}+\zeta_{ \pm}\right)}{\left(G_{\mathrm{eff}}^{v}+\zeta_{ \pm}\right)}
$$

where

$$
\zeta_{ \pm}=\frac{G_{ \pm}}{6}\left(\frac{9 K_{ \pm}+8 G_{ \pm}}{K_{ \pm}+2 G_{ \pm}}\right)
$$

In (28), the values of $G_{ \pm}$and $K_{ \pm}$are those defined algorithmically according to:

$$
K_{ \pm}=\frac{K_{V}\left(G_{\mathrm{eff}}^{r}-G_{ \pm}\right)}{\left(G_{\mathrm{eff}}^{v}-G_{ \pm}\right)}
$$

where, for $K_{-}$,

$$
0 \leq G_{-} \leq \min \left(C_{44}, G_{\mathrm{eff}}^{r}, C_{66}\right)
$$

and, similarly, for the $K_{+}$formula,

$$
\max \left(C_{44}, G_{\mathrm{eff}}^{v}, C_{66}\right) \leq G_{+} \leq \infty
$$

The corresponding formulas for shear modulus bounds $G_{h e x}^{ \pm}$are

$$
\frac{1}{G_{\mathrm{hex}}^{ \pm}+\zeta_{ \pm}}=\frac{1}{5}\left[\frac{1-\alpha_{ \pm}\left(K_{V}-K_{ \pm}\right)}{G_{\mathrm{eff}}^{v}+\zeta_{ \pm}+\frac{\alpha_{ \pm}}{2 \beta_{ \pm}}\left(K_{V}-K_{ \pm}\right)}+\frac{2}{C_{44}+\zeta_{ \pm}}+\frac{2}{C_{66}+\zeta_{ \pm}}\right]
$$

where the constants $\alpha_{ \pm}$and $\beta_{ \pm}$are defined by

$$
\alpha_{ \pm}=\frac{-1}{K_{ \pm}+4 G_{ \pm} / 3}, \quad \beta_{ \pm}=\frac{2 \alpha_{ \pm}}{15}-\frac{1}{5 G_{ \pm}} .
$$

Peselnick and Meister [25] had originally obtained all the results for hexagonal symmetry, except for an additional condition that permits $c_{44}$ to be replaced in some circumstances by $G_{\text {eff }}^{r}$. This condition was added later by Watt and Peselnick [26]. 


\section{Self-consistent Estimates for Hexagonal Symmetry}

The results obtained for self-consistent estimates can be written in many different ways [6]. We take the self-consistent estimate for bulk modulus to be

$$
K^{*}=\frac{K_{V}\left(G_{\mathrm{eff}}^{r}+\zeta^{*}\right)}{\left(G_{\mathrm{eff}}^{v}+\zeta^{*}\right)}=\frac{\left(G_{\mathrm{eff}}^{v} K_{R}+\zeta^{*} K_{V}\right)}{\left(G_{\mathrm{eff}}^{v}+\zeta^{*}\right)},
$$

where

$$
\zeta^{*}=\frac{G^{*}}{6}\left(\frac{9 K^{*}+8 G^{*}}{K^{*}+2 G^{*}}\right) .
$$

In $(35), K^{*}$ is determined by $(34)$, depending also on $G^{*} ; G^{*}$ is determined by the selfconsistent expression for the shear modulus to follow, also depending on $K^{*}$; and $\zeta^{*}$ is then determined by (35). The final result for $G^{*}=G_{\text {hex }}^{*}$ in polycrystals having grains with hexagonal symmetry is

$$
\frac{1}{G_{\mathrm{hex}}^{*}+\zeta^{*}}=\frac{1}{5}\left[\frac{1-\alpha^{*}\left(K_{V}-K^{*}\right)}{G_{\mathrm{eff}}^{v}+\zeta^{*}}+\frac{2}{C_{44}+\zeta^{*}}+\frac{2}{C_{66}+\zeta^{*}}\right] .
$$

These formulas can be successfully solved by iteration, starting for example by using values

corresponding to upper or lower bounds for the values of $K^{*}$ and $G^{*}$. Some details of the derivation of these formulas can be found in Willis [13, 14] and Berryman [6].

\section{Appendix B: Crack-influence Decomposition Method}

Sayers and Kachanov [5] present a useful method for decomposing the elastic potential of a cracked system into parts due to the (assumed) homogeneous and isotropic elastic background material, and those due to the presence of cracks up to moderate densities. The fundamental idea is that the elastic potential function is composed of just nine terms,

representing all combinations of stress invariants of such a system. These invariants depend on the stress tensor $\sigma$ and the crack density tensor $\alpha$. In particular, the tensor $\alpha$ is defined in three dimensions by

$$
\alpha=\frac{1}{V} \sum_{c} a_{c}^{3} \hat{n}_{c} \hat{n}_{c}^{T}
$$

where $V$ is the averaging volume, $\hat{n}_{c}$ is the unit normal of penny-shaped crack $c$ having radius $a_{c}$. We use the notation $\hat{n}_{c} \hat{n}_{c}^{T}$, where $T$ is the transpose, to express the outer product $\left(\hat{n}_{c} \otimes \hat{n}_{c}\right)$ of two vectors; this notation is consistent with that commonly used to express the singular value decomposition of an arbitrary matrix in terms of its singular vectors. Another 
common, and entirely equivalent, form of notation for the same quantity that is often used in the mechanics literature is the dyadic form $\boldsymbol{n}_{c} \boldsymbol{n}_{c}$.

The elastic potential $\Phi(\sigma, \alpha)$ then takes the form

$$
\begin{aligned}
\Phi(\sigma, \alpha) & =\Phi_{1}^{(0)}(\operatorname{Tr} \sigma)^{2}+\Phi_{2}^{(0)} \operatorname{Tr}(\sigma \cdot \sigma) & +\eta_{1} \operatorname{Tr} \sigma \operatorname{Tr}(\sigma \cdot \alpha) \quad+\eta_{2} \operatorname{Tr}(\sigma \cdot \sigma \cdot \alpha) \\
& +\eta_{3}[\operatorname{Tr}(\sigma \cdot \alpha)]^{2} & +\eta_{4} \operatorname{Tr} \sigma \operatorname{Tr}(\sigma \cdot \alpha \cdot \alpha)+\eta_{5} \operatorname{Tr}(\sigma \cdot \sigma \cdot \alpha \cdot \alpha) \\
& +\eta_{6} \operatorname{Tr}(\sigma \cdot \alpha) \operatorname{Tr}(\sigma \cdot \alpha \cdot \alpha) & +\eta_{7}[\operatorname{Tr}(\sigma \cdot \alpha \cdot \alpha)]^{2},
\end{aligned}
$$

where $\operatorname{Tr}$ is the trace operation, and the dot notation indicates a contraction over one set of indices. (Note that the significance of crack-influence parameters $\eta_{4}, \eta_{6}$, and $\eta_{7}$ have been changed from the definitions made by Kachanov [27], Kachanov and Sevostianov [28], and Sayers and Kachanov [5], so that here $\eta_{4}$ is the coefficient of a contribution second order in $\alpha, \eta_{6}$ third order in $\alpha$, and $\eta_{7}$ fourth order in $\alpha$.) The coefficients pertinent to the isotropic background elastic medium are given by $\Phi_{1}^{(0)}=\left(1+\nu_{0}\right) / 2 E_{0}$ and $\Phi_{2}^{(0)}=-\nu_{0} / 2 E_{0}$, where $E_{0}$ is Young's modulus, and $\nu_{0}$ is Poisson's ratio.

Now, to illustrate the meaning of (38), we will reduce this to component form in two cases. For the cases of interest, we can assume the crack density tensor itself reduces to the form

$$
\alpha=\sum_{i=1}^{3} \rho_{i} \hat{n}_{i} \hat{n}_{i}^{T},
$$

where $\hat{n}_{i}$, for $i=1,2,3$, correspond to spatial directions $x, y, z$, respectively. Furthermore, $\operatorname{Tr} \alpha=\rho_{1}+\rho_{2}+\rho_{3} \equiv \rho=n a^{3}$ is the scalar crack density defined in the main text.

\section{Horizontal cracks, $\rho=\rho_{3}$}

If all the cracks in the system have the same axis of symmetry (which we will take to be the $z$-axis), then $\rho=\rho_{3}$ and (38) reduces to the following expression:

$$
\begin{gathered}
\Phi(\sigma, \alpha)=\Phi_{1}^{(0)}(\operatorname{Tr} \sigma)^{2}+\Phi_{2}^{(0)} \operatorname{Tr}(\sigma \cdot \sigma)+\left(\eta_{1} \rho+\eta_{4} \rho^{2}\right)\left(\sigma_{11}+\sigma_{22}+\sigma_{33}\right) \sigma_{33} \\
+\left(\eta_{2} \rho+\eta_{5} \rho^{2}\right) \sigma_{3 j} \sigma_{j 3}+\left(\eta_{3} \rho^{2}+\eta_{6} \rho^{3}+\eta_{7} \rho^{4}\right) \sigma_{33}^{2}
\end{gathered}
$$

where the repeated index $j$ is summed. At low crack densities $\rho$, we see that only the terms proportional to $\eta_{1}$ and $\eta_{2}$ are important in the crack-influence decomposition. As the crack density increases, the terms proportional to $\eta_{3}, \eta_{4}$, and $\eta_{5}$ start to contribute. Then, at the 
highest crack densities considered, all seven of these coefficients can come into play. Although we may imagine for example that $\eta_{2}(\rho)$ is actually a function of crack density $\rho$, it is clear from the form of (40) that such corrections would be indistinguishable from corrections due to $\eta_{5}(0)$. So, for our present purposes, we do not need to consider any coefficients except $\eta_{1}(0)$ and $\eta_{2}(0)$ at low crack densities, and we also do not need to consider any coefficients except $\eta_{1}(0)$ through $\eta_{5}(0)$, when we want to fit quadratic corrections for the moderate crack density results.

Typical values of $\rho$ of interest in many applications are around $\rho=0.1$. So as long as the $\eta$ 's for higher order corrections are of approximately the same order of magnitude as those for $\eta_{1}(0)$ and $\eta_{2}(0)$, we see that neglect of terms like $\eta_{6} \rho^{3}+\eta_{7} \rho^{4}$ is entirely appropriate.

Now it is also easy to see how (40) gives rise to the low density result (4).

\section{Vertical cracks, $\rho=\rho_{1}+\rho_{2}$}

It is also straightforward now to repeat the previous exercise by considering other types of crack density distribution. An interesting case is the one with all vertical cracks, having their crack normals in the $x y$-plane. Then, $\rho=\rho_{1}+\rho_{2}$. A special case of this type is when the crack normals are completely randomly distributed so that $\rho_{1}=\rho_{2}=\rho / 2$. Then, we get simplified formulas for all the terms in the elastic potential analogous to the previous example. The results are:

$$
\begin{aligned}
& \Phi(\sigma, \alpha)=\Phi_{1}^{(0)}(\operatorname{Tr} \sigma)^{2}+\Phi_{2}^{(0)} \operatorname{Tr}(\sigma \cdot \sigma)+\left(\eta_{1} \rho / 2+\eta_{4} \rho^{2} / 4\right)\left(\sigma_{11}+\sigma_{22}+\sigma_{33}\right)\left(\sigma_{11}+\sigma_{22}\right) \\
& +\left(\eta_{2} \rho / 2+\eta_{5} \rho^{2} / 4\right)\left(\sigma_{1 j} \sigma_{j 1}+\sigma_{2 j} \sigma_{j 2}\right)+\left(\eta_{3} \rho^{2} / 4+\eta_{6} \rho^{3} / 8+\eta_{7} \rho^{4} / 16\right)\left(\sigma_{11}+\sigma_{22}\right)^{2}
\end{aligned}
$$

where again the repeated index $j$ is summed.

The basic conclusions reached in the previous example clearly apply again. For small to moderate crack densities $\rho$, we do not need to consider $\rho$ dependence of crack-influence parameters $\eta_{1}(\rho)$ or $\eta_{2}(\rho)$, as such dependence cannot be distinguished from the low order contributions from $\eta_{4}(0)$ and $\eta_{5}(0)$, respectively. Similarly, $\eta_{3}(0)$ comes into play whenever $\eta_{4}(0)$ and $\eta_{5}(0)$ are important, while $\eta_{6}(0)$ and $\eta_{7}(0)$ can presumably be neglected in many low to moderate crack density applications. 
TABLE 2. Examples of Sayers and Kachanov [5] crack-influence parameters $\eta_{1}(\rho)$ and $\eta_{2}(\rho)$ when crack density $\rho<<1$ for penny-shaped cracks. Four choices of effective medium theory are considered: NI (non-inteacting), DS, (differential scheme), CPA (coherent potential approximation), and SC (the Budiansky and O'Connell self-consistent scheme). Note that crack density is defined here as $\rho=N r^{3} / V$, where $N / V$ is number density of cracks, and $A=\pi r^{2}$ is the area of the circular crack face.

\begin{tabular}{|c|cc|}
\hline \hline & $\eta_{1}\left(\mathrm{GPa}^{-1}\right)$ & $\eta_{2}\left(\mathrm{GPa}^{-1}\right)$ \\
\hline $\mathrm{NI}$ & -0.000216 & 0.0287 \\
$\mathrm{DS}$ & -0.000216 & 0.0290 \\
$\mathrm{CPA}$ & -0.000258 & 0.0290 \\
$\mathrm{SC}$ & -0.0000207 & 0.0290 \\
\hline \hline
\end{tabular}

\section{APPENDIX C. DISCUSSION OF EFFECTIVE MEDIUM THEORIES}

To place our method using random polycrystal analysis in perspective, we will now give a brief review and discussion of other effective medium theories that have often been applied to the same types of crack-related problems treated here.

A very good review of many (but not all) of the effective medium theories we will mention here has already been given by Zimmerman [18]. To keep the present discussion as brief as possible, we will just provide an overview here, but no equations. In Zimmerman's treatment of the methods, he does include the equations and therefore much of what is missing here can be found in reference [18], and the remainder is found in the other references given.

One of the main advantages of the random polycrystals of cracked-grains model is that it provides a definite means of building in a type of microstructure that is usually not possible to obtain using other effective medium theories. This microstructure arises naturally in the polycrystal analysis because we must first construct a grain, using an initial estimate of the effect of the crack (or cluster of cracks) on the grain. Then, we imagine jumbling these cracked grains together to arrive at the overall polycrystalline microstructure. So this approach involves two distinct steps of upscaling: first at the grain level, and then for the overall fractured composite.

The presence of two, or even many, steps of upscaling in effective medium theories is 
not at all unusual. The best examples of this are the differential schemes [18, 21, 29-32] in which we take a pristine elastic medium and imbed a very small amount of some inclusion (or a small crack in our case). Then, we use an integration method to deduce what the overall effect of this small volume or small crack density will be on the overall medium. The stated procedure is already one level of upscaling. Then, we do this over and over again in the differential scheme, each time starting from the result of the last upscaling step. So small quantities of new inclusions or cracks get imbedded in a medium that already has inclusions or cracks, and so on. Certain of these differential schemes are realizable [31, 33], and therefore never violate rigorous bounds.

In contrast, traditional self-consistent schemes [34] and also more modern types of selfconsistent scheme that are some times termed the "coherent potential approximation" (or CPA) [35] also achieve their final results using multiple steps of upscaling, but this happens at a fixed target volume fraction through an iterative method: we start with coupled equations that depend on constituent properties, volume fractions, and also on the overall properties (usually overall bulk and shear modulus); then these equations are provided with some initial guess of the overall property values, and subsequently iterated until they converge to a definite result. This iteration process itself can be viewed as being very similar to the differential scheme in the sense that each new iteration is using the result of previous (approximate) upscaling steps to generate the next upscaling, until final convergence is achieved. We can also think of this procedure as providing a type of scale-separation at each iteration [36].

One important difference between these two schemes (differential and self-consistent) is that the differential scheme usually starts with one material as the host, and so that host remains connected throughout the integration process. In contrast, the self-consistent scheme treats two or more constituents equally, with no one of them necessarily playing the role of host (but of course if one has significantly higher volume fraction than all the others, then a host-inclusion type of microstructure will necessarily arise).

In addition to these implicit schemes (requiring integration or iteration), there are also some explicit schemes: Mori-Tanaka [37], Kuster-Toksöz [38], and the non-interaction approximation [18]. Explicit schemes provide formulas: needing numerical evaluation, but not needing either integration or iteration. However, these schemes are known not to be so reliable for very high concentrations of inclusions, and, furthermore, they can lead to incorrect 
results, such as violations of known rigorous bounds such as the Hashin-Shtrikman bounds when the inclusion shapes are extreme (i.e., differing greatly from spheres). So care must be exercised when using these methods ( - and also the other methods as well). But the CPA [36] and the differential scheme due to Norris [31, 33] are known not to violate the Hashin-Shtrikman bounds, and therefore tend to be fairly reliable estimators, depending on the application and the particular microstructure that one is trying to emulate.

So, among all of the effective medium theories mentioned, only the explicit schemes use just a single upscaling step to arrive at their elastic constant estimates, and this one step may not be trustworthy if the volume fractions of the inclusions, or crack-density of the cracks, is too large [39].

In TABLE 2 we show the results found by applying the non-interaction (NI) approximation, the Norris differential scheme (DS), the coherent potential approximation (CPA) and the traditional self-consistent (SC) scheme as applied by O'Connell and Budiansky [34]. We find (as expected) that all these methods give very comparable results for the Sayers and Kachanov [5] crack-influence decomposition parameters $\eta_{1}$ and $\eta_{2}$ at low crack densities. These results suggest that it is entirely appropriate to use the NIA when making the first upscaling step for estimating the properties of a typical cracked grain.

The method proposed here for the random polycrystal of cracked-grains model is then a two-step process: The first step has been chosen to be a non-interaction approximation based on low crack density results from the theory. This step gives us the effective elastic behavior of an average cracked grain. Once we have this compliance in hand, our second upscaling step uses the polycrystal analysis to provide Voigt and Reuss bounds, and also self-consistent estimates. We show further results for Hashin-Shtrikman bounds in the figures in the text, but unfortunately these results show that HS bounds are too tight for these applications, and therefore it is inferred that assumptions implicit in the derivation of HS bounds have been violated. Note that the second step involving Voigt and Reuss bounds is also entirely explicit, while the alternative second step involving the self-consistent estimates for polycrystals is implicit (requiring iteration). So the random polycrystal bounding approach should be very appealing to those users in particular who greatly prefer explicit methods. 


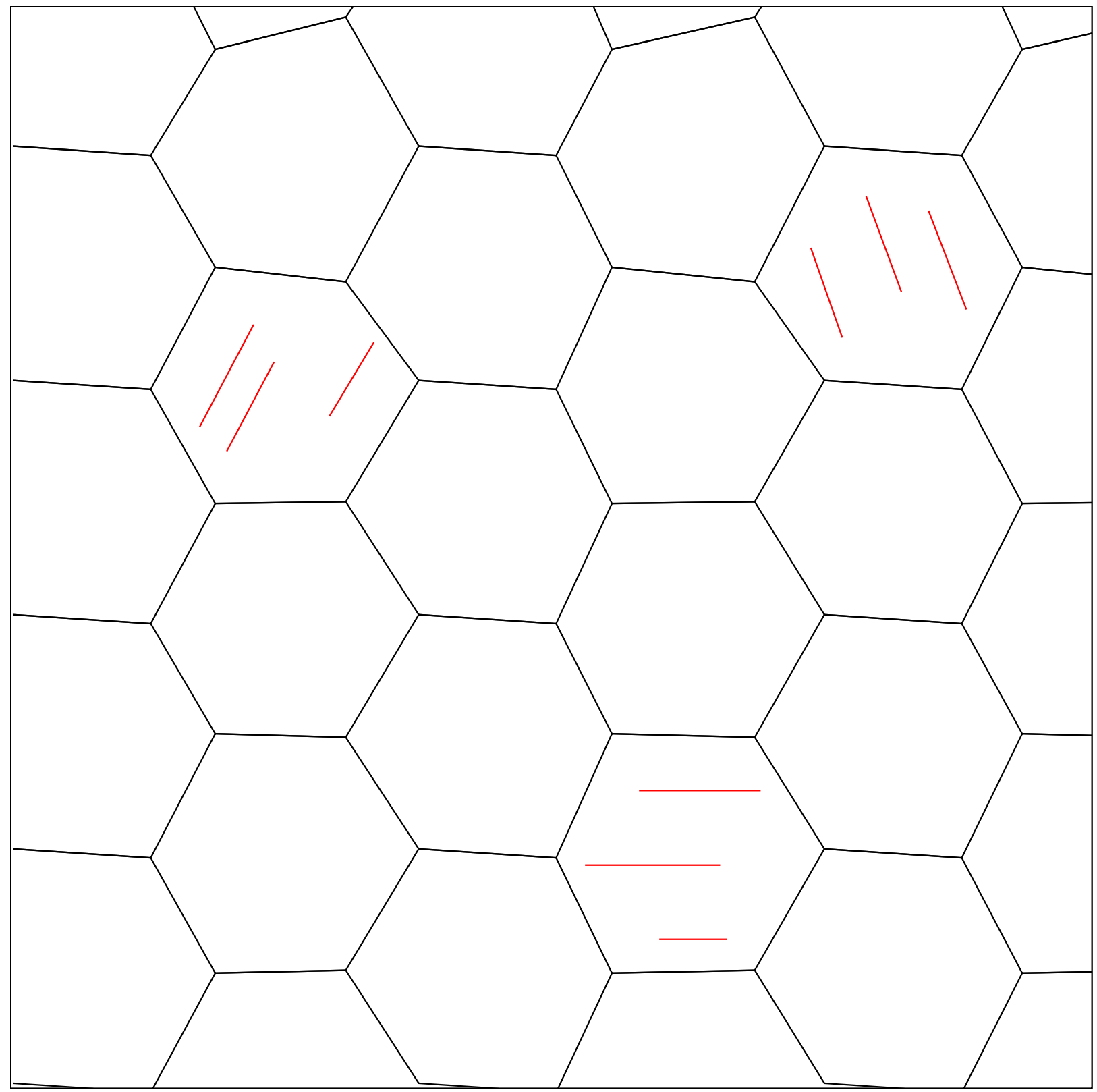

FIG. 1: Schematic illustrating the random polycrystals of cracked grains model. Grains are assumed to fit tightly so there is no misfit porosity, although there is some porosity due to the cracks themselves. The shapes of the grains are not necessarily the same, and the symmetry axes of the grains (three examples shown) are randomly oriented so the overall polycrystal is equiaxed (statistically isotropic). 


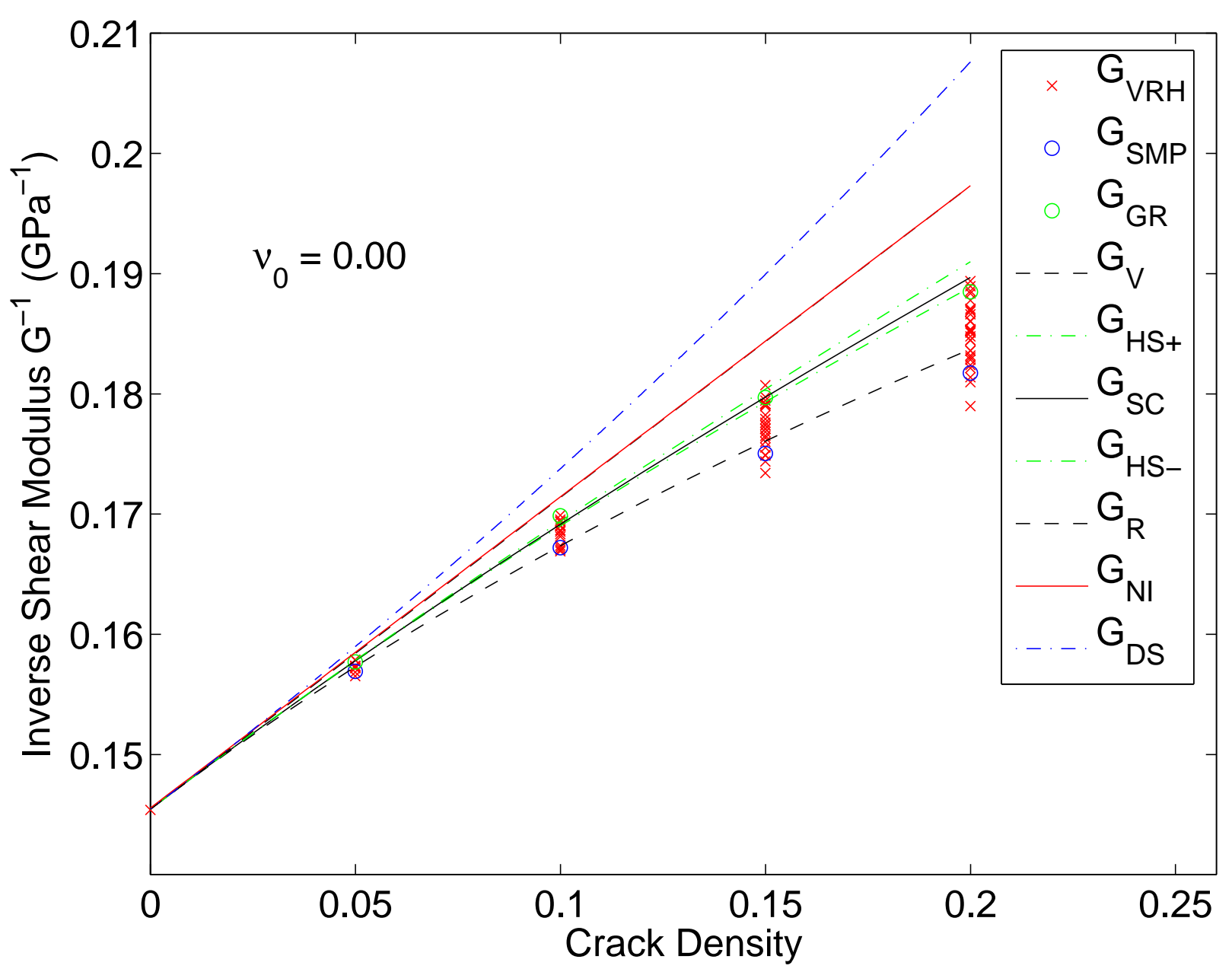

FIG. 2: Estimates (SC, NI, and DS) and possible bounds (R, $\left.\mathrm{HS}^{-}, \mathrm{HS}^{+}, \mathrm{V}\right)$ on inverse shear modulus estimators $\mathrm{G}_{V R H}^{-1}$ of 33 examples from the numerical experiments of Grechka and Kachanov. Background medium has Poisson's ratio $\nu_{0}=0.0$ for this example. Crack density $\rho=n a^{3}$, where $n=$ number of penny-shaped cracks per unit volume, and $a$ is the radius of the cracks. (If the cracks are not all the same size, then the product $n a^{3}$ is the appropriate average quantity.) Crack aspect ratio is assumed small, but nonzero. The estimates of individual (cracked) grain behavior as crack density increases make use of crack-influence parameters $\eta_{1}=0.0 \mathrm{GPa}^{-1}$ and $\eta_{2}=0.194448$ $\mathrm{GPa}^{-1}$ (see Sayers and Kachanov [5] for definitions) determined using the DS estimator for small crack densities. Similarly, for the NI estimator: $\eta_{1}=0.0 \mathrm{GPa}^{-1}$ and $\eta_{2}=0.1941 \mathrm{GPa}^{-1}$. Note that the estimates and bounds are all consistently high (for $G^{-1}$ ) compared to the numerical data (x). Polycrystal bounds and estimates were obtained here without any fitting parameters, using only the very low crack density coefficients, $\eta_{1}$ and $\eta_{2}$. 


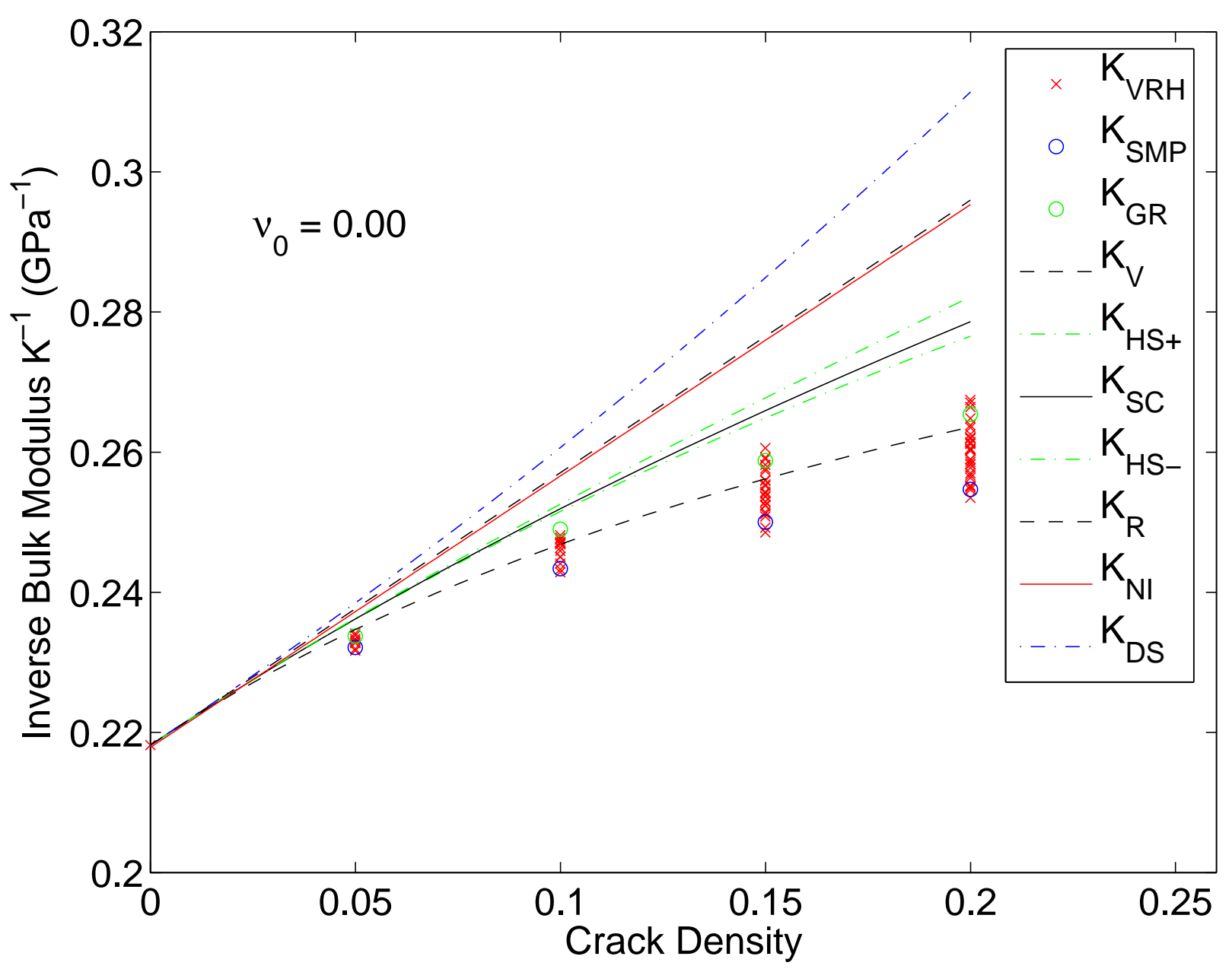

FIG. 3: Same as Figure 2, showing corresponding results for the inverse bulk modulus estimators $\mathrm{K}_{V R H}^{-1}$. Polycrystal bounds and estimates were also obtained here without any fitting parameters, using only the very low crack density coefficients. Note that the estimates and possible bounds are considerably higher here in relation to the numerical data $(\mathrm{x})$ than in Figure 2 for the inverse shear modulus. We interpret this difference as being a result of shorter range interactions for shear, and longer range interactions for bulk modulus that are not properly taken into account by the present (overly simple) model. 


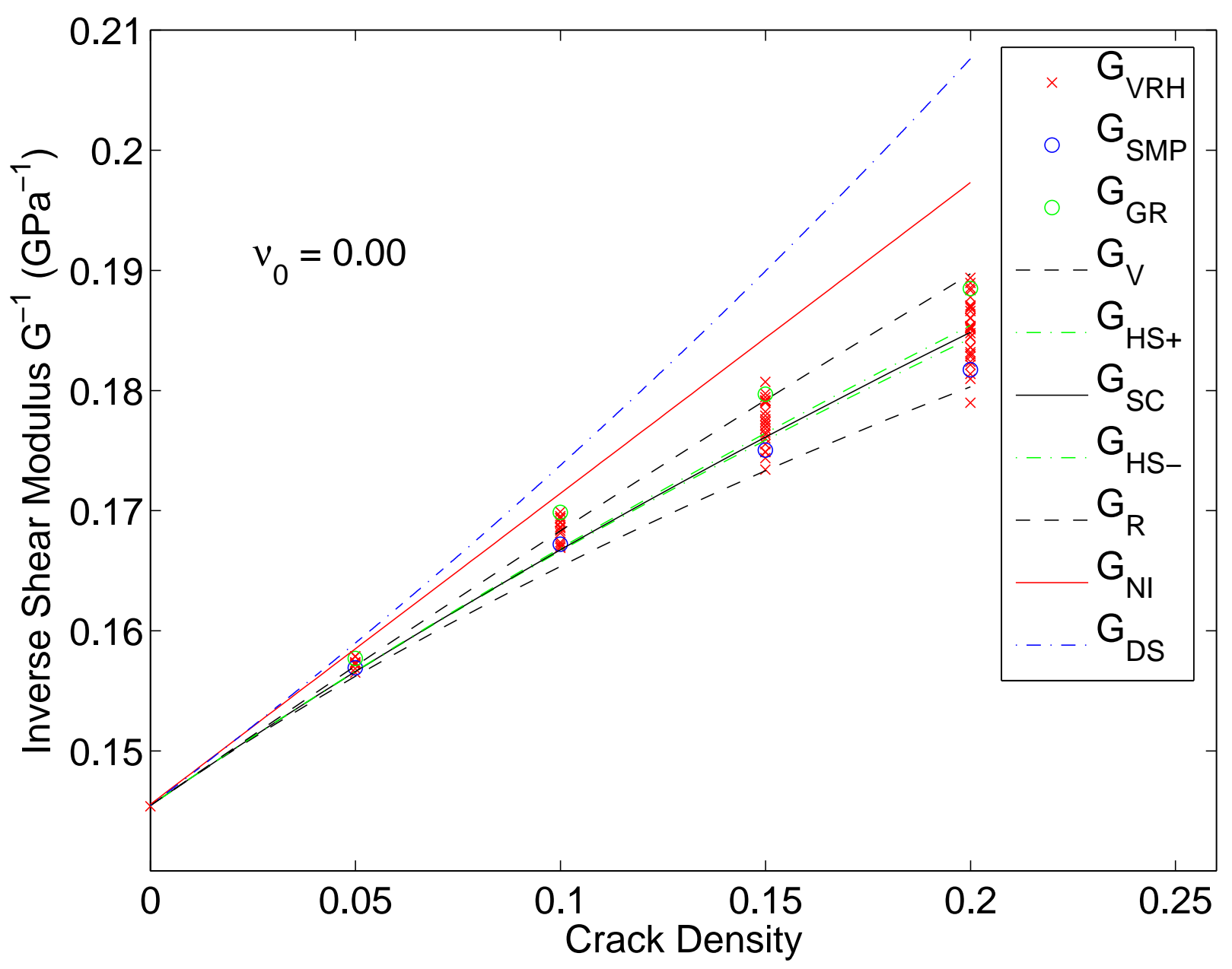

FIG. 4: Same as Figure 2, but the values of $\eta_{1}$ and $\eta_{2}$ were obtained from an average of the shear modulus data $(\mathrm{x})$ at $\rho=0.05$. Furthermore, a quadratic correction is added to compliance values $S_{33}, S_{44}$, and $S_{55}$ to give the best fit both here and simultaneously in Figure 5 . Note that the spread in the data is comparable to the difference in the Reuss and Voigt bounds, but substantially greater than the spread in the HS bounds. 


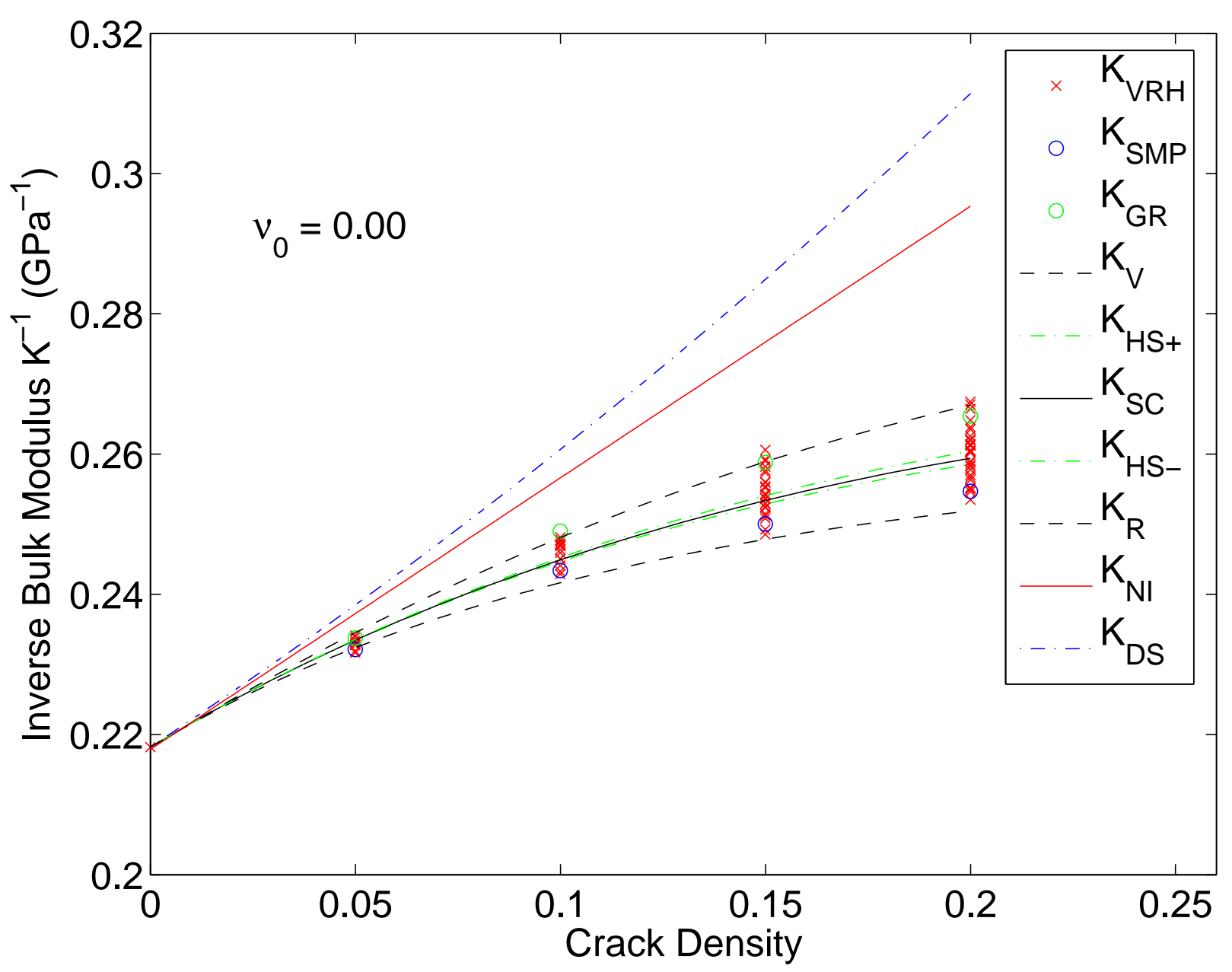

FIG. 5: Same as Figure 4, for the inverse bulk modulus. Note that the data mostly fall between the Reuss and Voigt bounds, but definitely are not close to being restricted to lie inside the HS bounds. 


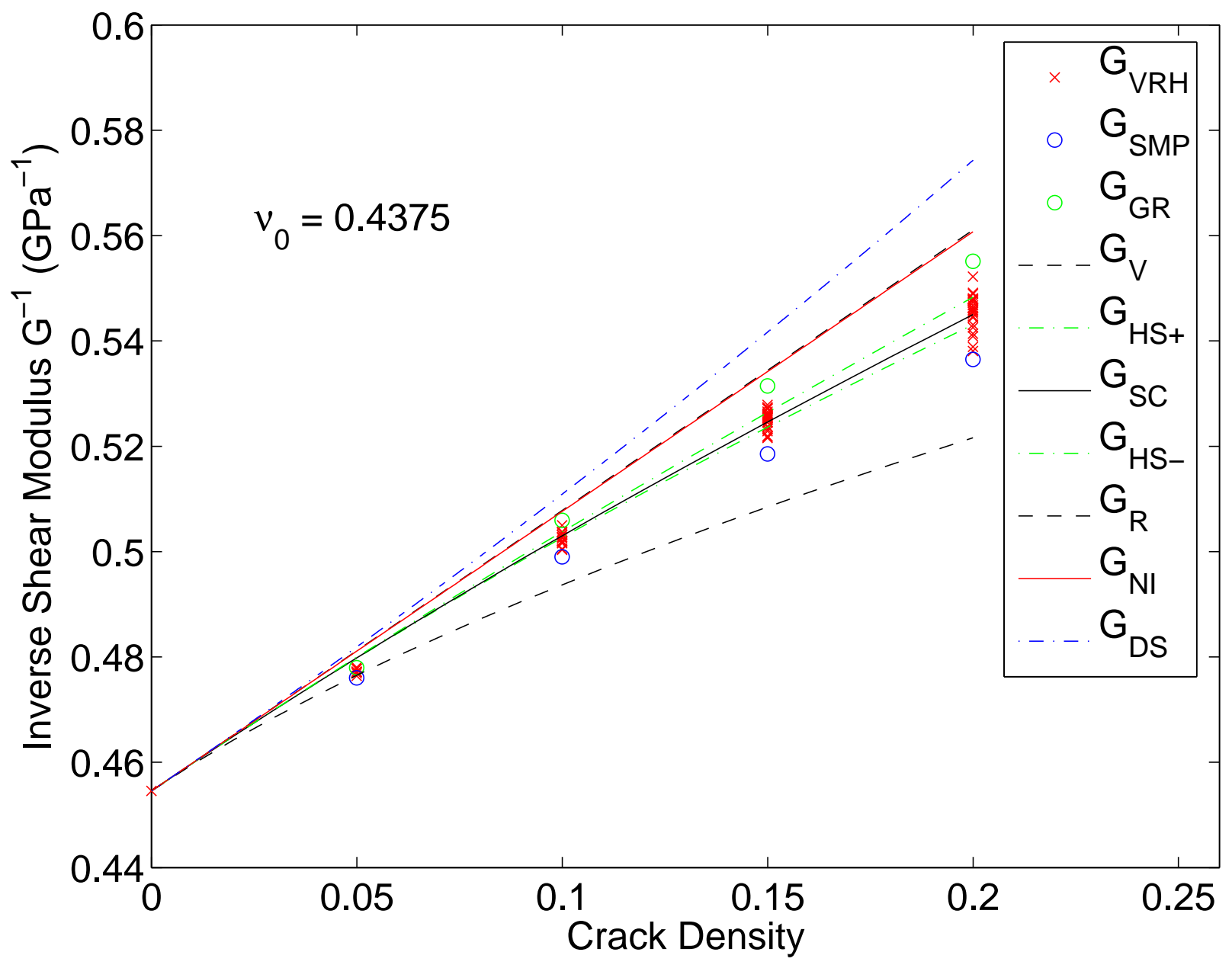

FIG. 6: Same as Figure 2 for a different background medium having Poisson's ratio $\nu_{0}=0.4375$. The estimates of individual grain behavior as crack density increases make use of $\eta_{1}=-0.0191973$ $\mathrm{GPa}^{-1}$ and $\eta_{2}=0.399406 \mathrm{GPa}^{-1}$ (see Sayers and Kachanov [5] for definitions of the $\eta$ 's) determined using the DS estimator for small crack densities. Similarly, for the NI estimator: $\eta_{1}=-0.0191$ $\mathrm{GPa}^{-1}$ and $\eta_{2}=0.3982 \mathrm{GPa}^{-1}$. Note that, again without any fitting, the estimates of inverse shear modulus are quite accurate for this model. 


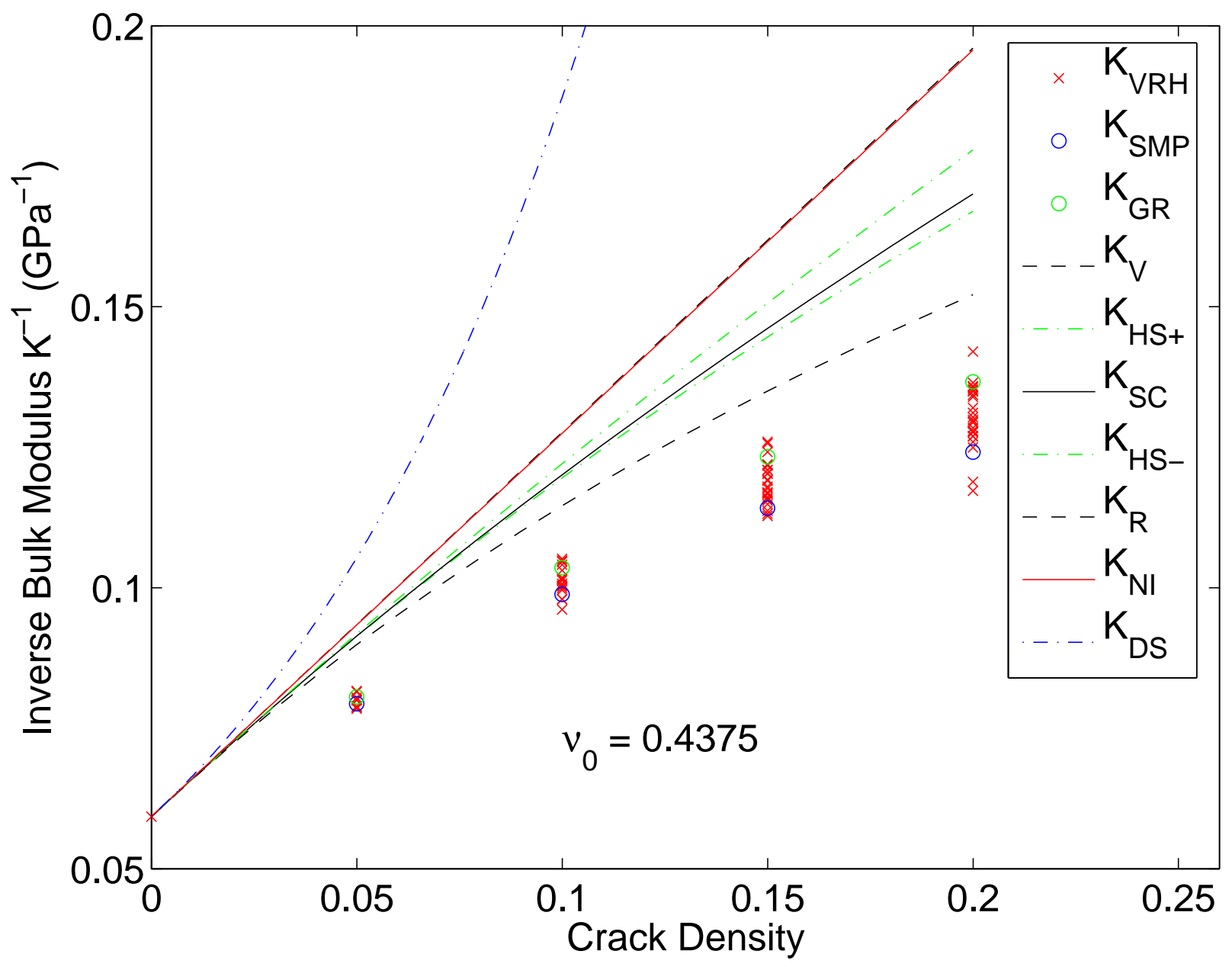

FIG. 7: Same as Figure 6 for inverse bulk modulus. Note that the estimates are again quite high for the inverse bulk modulus when compared to the numerical data. This result is in contrast to the shear modulus example in Figure 6, where the initial estimates were very close to the numerical data, both in average and spread. 


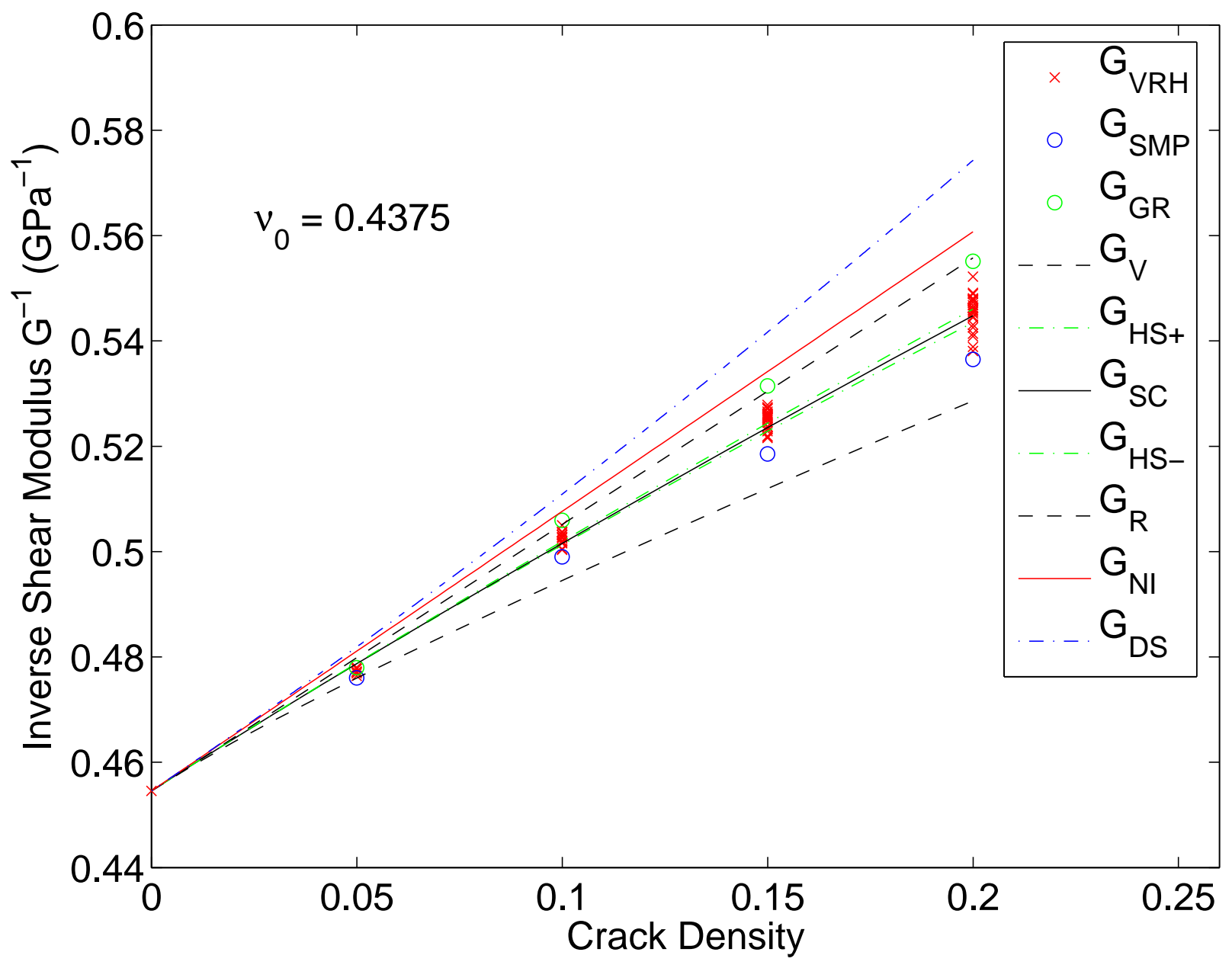

FIG. 8: Same as Figure 4 for the case with $\nu_{0}=0.4375$. In this case, the spread in the data is smaller than the difference in the Reuss and Voigt bounds, but again significantly greater than that of the HS bounds. 


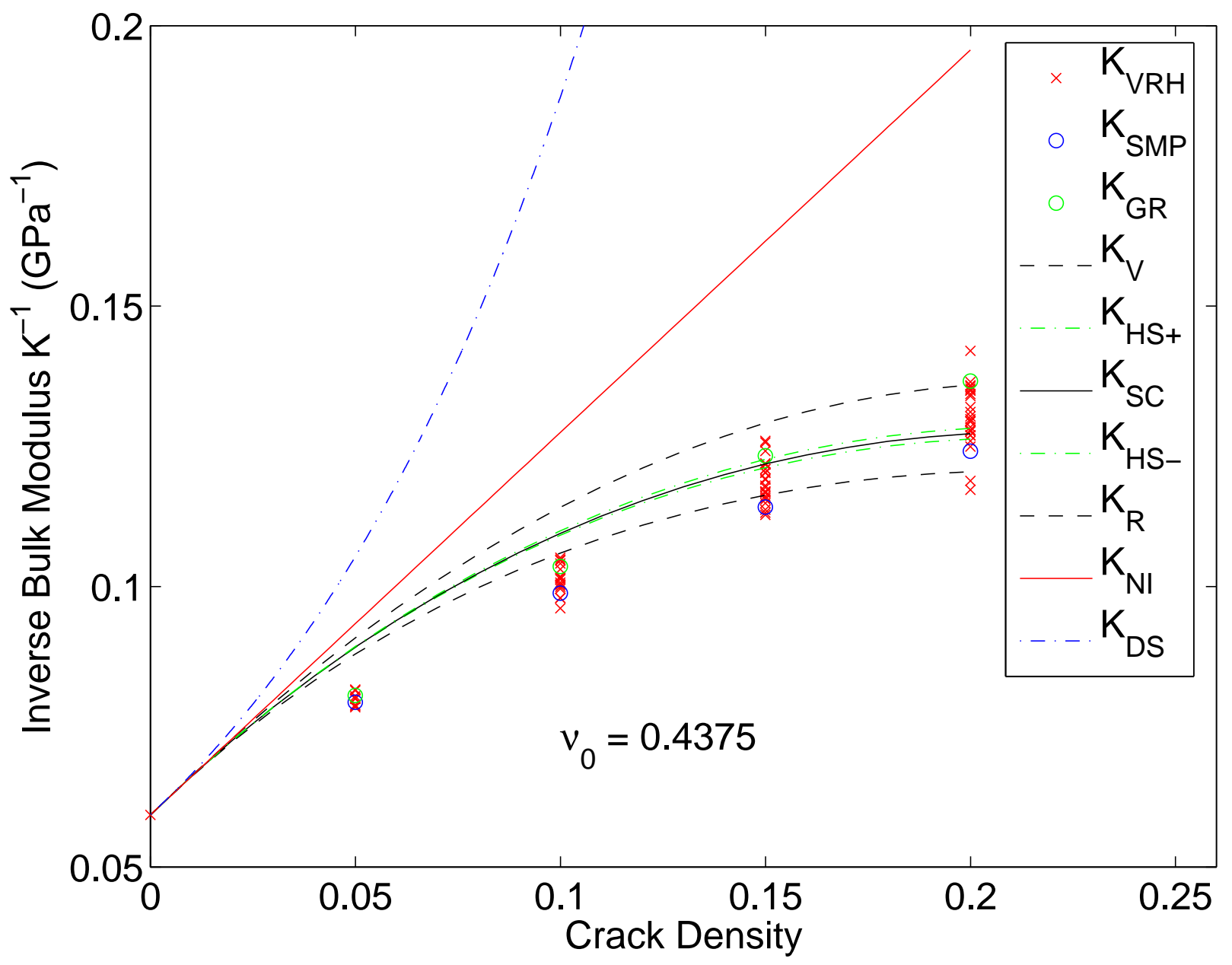

FIG. 9: Same as Figure 5 for the case with $\nu_{0}=0.4375$. Spread in the inverse bulk modulus values (x) is again comparable to that between the Reuss and Voigt bounds. But some of the numerical data at $\rho=0.05$ and $\rho=0.10$ could not be fit for this example using the simple quadratic corrections described in the text. 